\title{
Eigenfunctions of Linear Canonical Transform
}

\author{
Soo-Chang Pei, Fellow, IEEE, and Jian-Jiun Ding
}

\begin{abstract}
The linear canonical transform (the LCT) is a useful tool for optical system analysis and signal processing. It is parameterized by a $2 \times 2$ matrix $\{a, b, c, d\}$. Many operations, such as the Fourier transform (FT), fractional Fourier transform (FRFT), Fresnel transform, and scaling operations are all the special cases of the LCT. In this paper, we will discuss the eigenfunctions of the LCT. The eigenfunctions of the FT, FRFT, Fresnel transform, and scaling operations have been known, and we will derive the eigenfunctions of the LCT based on the eigenfunctions of these operations. We find, for different cases, that the eigenfunctions of the LCT also have different forms. When $|a+d|<2$, the eigenfunctions are the scaling, chirp multiplication of Hermite functions, but when $|a+d|=2$, the eigenfunctions become the chirp multiplication, chirp convolution of almost-periodic functions (see Section IV-C), or impulse trains. In addition, when $|a+d|>2$, the eigenfunctions become the chirp multiplication and chirp convolution of self-similar functions (fractals). Besides, since many optical systems can be represented by the LCT, we can thus use the eigenfunctions of the LCT derived in this paper to discuss the self-imaging phenomena in optics. We will show that there are usually varieties of input functions that can cause the self-imaging phenomena for an optical system.
\end{abstract}

Index Terms-Fourier transform, fractional Fourier transform, Fresnel transform, linear canonical transform.

\section{INTRODUCTION}

$\mathbf{T}$ HE LINEAR canonical transform (the LCT) [1]-[4] is an integral transform with four parameters $\{a, b, c, d\}$. It was first introduced in 1970s [5], [6]. Many operations, such as the Fourier transform (FT), fractional Fourier transform (FRFT) [7]-[9], Fresnel transform [10], and scaling operations are the special cases of the LCT. In some papers, the LCT is also called as the affine Fourier transform (AFT) [2], [11], the generalized Fresnel transform [12], the Collins formula [6], the ABCD transform [3], or the almost Fourier and almost Fresnel transformation [4]. The LCT is useful for optics because many optical systems can be represented by the LCT [13]-[15]. Besides, the LCT is also useful for radar system analysis, GRIN medium system analysis, filter design, phase retrieval, pattern recognition, and many other applications.

In this paper, we will derive the eigenfunctions of the LCT.

The eigenfunctions of some special cases of the LCT have been known. For example, the eigenfunctions of the FRFT have been known as the Hermite functions multiplied by $\exp \left(-t^{2} / 2\right)$ [see (17)]. The eigenfunctions of the LCT when $\{a, b, c, d\}=\{1, b, 0,1\}$ (in this case, the LCT becomes the Fresnel transform) are known as the periodic functions (this

Manuscript received February 6, 2001; revised September 28, 2001. The associate editor coordinating the review of this paper and approving it for publication was Prof. Fredrik Gustafsson.

The authors are with the Department of Electrical Engineering, National Taiwan University, Taipei, Taiwan, R.O.C (e-mail: pei@cc.ee.ntu.edu.tw).

Publisher Item Identifier S 1053-587X(02)00413-0. is called the Talbot effect [16], [17]). For the case where $\{a, b, c, d\}=\{1 / d, 0,0, d\}$ (in this case, the LCT becomes the scaling operation), the eigenfunctions are known as the self-similar functions [18], [19] (i.e., fractals). These functions are invariant after doing the scaling operations. Besides, in [12], they have already derived the eigenfunctions of the LCT for the case where $|a+d|<2$.

In this paper, we will improve and generalize the previous works and derive the eigenfunctions of the LCT for all the other cases. After the eigenfunctions of the LCT have been derived, we can use them to analyze the self-imaging phenomena in optics.

Our paper is organized as follows. First, in Section II, we will review the FRFT and the LCT as well as the previous works about the eigenfunctions of FRFT and the LCT. In Section III, we discuss the eigenfunctions of the LCT for the case in which $|a+d|<2$ and improve the works in [12]. In Section IV, we will derive the eigenfunctions of the LCT for the case in which $|a+d|=2$ based on the eigenfunctions of chirp convolution operation (i.e., impulse trains). In Section V, we derive the eigenfunctions of the LCT for the case in which $|a+d|>2$ based on the scaling invariant functions (fractals). Then, with the aid of the eigenfunctions of the LCT derived in Sections III-V, in Section VI, we will discuss the self-imaging phenomena in optics. In addiiton, in Section VII, we summarize the eigenfunctions of the LCT we derived and make a conclusion.

\section{PReliminary AND PRevious Works}

\section{A. Linear Canonical Transform and its Special Cases}

The linear canonical transform (the LCT) [1]-[3] is defined as

$$
\text { •the LCT } \begin{aligned}
& O_{F}^{(a, b, c, d)}(f(t)) \\
&= \sqrt{\frac{1}{j 2 \pi b}} e^{(j / 2)(d / b) u^{2}} \\
& \cdot \int_{-\infty}^{\infty} e^{-j(u / b) t} e^{(j / 2)(a / b) t^{2}} f(t) d t \\
& \text { for } b \neq 0 \\
& O_{F}^{(a, b, c, d)}(f(t))=\sqrt{d} \cdot e^{(j / 2) c d u^{2}} f(d \cdot u) \\
& \text { for } b=0
\end{aligned}
$$

where

$$
a d-b c=1 .
$$

It satisfies the additivity property

$$
O_{F}^{\left(a_{2}, b_{2}, c_{2}, d_{2}\right)}\left(O_{F}^{\left(a_{1}, b_{1}, c_{1}, d_{1}\right)}(f(t))\right)=O_{F}^{\left(a_{3}, b_{3}, c_{3}, d_{3}\right)}(f(t))
$$


where

$$
\left[\begin{array}{ll}
a_{3} & b_{3} \\
c_{3} & d_{3}
\end{array}\right]=\left[\begin{array}{ll}
a_{2} & b_{2} \\
c_{2} & d_{2}
\end{array}\right] \cdot\left[\begin{array}{ll}
a_{1} & b_{1} \\
c_{1} & d_{1}
\end{array}\right]
$$

Because the additivity property of the LCT can be described by the matrix operation as (5), we usually represent the LCT with parameters $\{a, b, c, d\}$ as the $2 \times 2$ matrix

$$
\left[\begin{array}{ll}
a & b \\
c & d
\end{array}\right]
$$

Many important operations are the special cases of the LCT. We describe them in the following.

\section{A) Fourier transform (FT):}

When $\{a, b, c, d\}=\{0,1,-1,0\}$, the LCT becomes the FT

$$
\begin{aligned}
\sqrt{j} \cdot O_{F}^{(0,1,-1,0)}(f(t)) & =\mathrm{FT}(f(t)) \\
& =\sqrt{\frac{1}{2 \pi}} \cdot \int_{-\infty}^{\infty} e^{-j \cdot u \cdot t} \cdot g(t) \cdot d t
\end{aligned}
$$

B) Fractional Fourier transform (FRFT):

The fractional Fourier transform (FRFT) [7]-[9] is defined as

-FRFT : $\quad O_{F}^{\alpha}(f(t))=\sqrt{\frac{1-j \cot \alpha}{2 \pi}} e^{(j / 2) \cot \alpha \cdot u^{2}}$

$$
\cdot \int_{-\infty}^{\infty} e^{-j \cdot \csc \alpha u t} e^{(j / 2) \cot \alpha t^{2}} f(t) d t
$$

It is the generalization of the FT. It satisfies the additivity property as

$$
O_{F}^{\alpha}\left(O_{F}^{\beta}(f(t))\right)=O_{F}^{\alpha+\beta}(f(t)) .
$$

The FRFT is useful for the applications of optical system analysis, filter design, solving differential equations, phase retrieval, pattern recognition, etc. Comparing (8) with (1), we find that the FRFT is the special case of the LCT where $\{a, b, c, d\}=\{\cos \alpha, \sin \alpha,-\sin \alpha, \cos \alpha\}$ and with some difference of constant phase

$$
O_{F}^{\alpha}(f(t))=\sqrt{e^{j \alpha}} \cdot O_{F}^{(\cos \alpha, \sin \alpha,-\sin \alpha, \cos \alpha)}(f(t)) .
$$

\section{C) Fresnel transform:}

The Fresnel transform is the operation that describes the monochromic light propagating through the free space [10]. It is defined as

- Fresnel transform: $O_{\text {Fresnel }}^{z}(f(x, y))=\frac{e^{j 2 \pi z / \lambda}}{j \lambda z}$

$$
\cdot \int_{-\infty}^{\infty} \int_{-\infty}^{\infty} e^{j(\pi / \lambda z)\left((u-x)^{2}+(v-y)^{2}\right)} f(x, y) d x d y
$$

where $f(x, y)$ is the distribution of the input monochromic light, $\lambda$ is its wavelength, and $z$ is the propagation distance. In fact, (11) also can be rewritten as the combination of two 1-D Fresnel transforms

$$
O_{\text {Fresnel }}^{z}(f(x, y))=O_{\text {Fresnel }(y)}^{z}\left(O_{\text {Fresnel }(x)}^{z}(f(x, y))\right)
$$

where

- 1-D Fresnel transform: $O_{\text {Fresnel }(x)}^{z}(g(x))=\frac{e^{j \pi z / \lambda}}{\sqrt{j \lambda z}}$

$$
\cdot \int_{-\infty}^{\infty} e^{j(\pi / \lambda z) \cdot(u-x)^{2}} g(x) \cdot d x
$$

Comparing (13) with (1), we find that the 1-D Fresnel transform is just the special case of the LCT when $\{a, b, c, d\}=\{1, b, 0,1\}$ with some difference of constant phase:

$$
O_{\text {Fresnel }(x)}^{z}(f(x))=e^{j \pi z / \lambda} \cdot O_{F}^{(1, z \lambda / 2 \pi, 0,1)}(f(x)) .
$$

The relation between the parameter $b$ and the propagation distance $z$ is

$$
b=\frac{z \lambda}{2 \pi} .
$$

\section{D) Scaling operation:}

Scaling can be viewed as the special case of the LCT when $\{a, b, c, d\}=\left\{\sigma^{-1}, 0,0, \sigma\right\}$ :

$$
O_{\mathrm{Sc}}^{\sigma}(g(t))=\sqrt{\operatorname{sgn}(\sigma)} \cdot O_{F}^{\left(\sigma^{-1}, 0,0, \sigma\right)}(g(t)) .
$$

Therefore, the FT, FRFT, Fresnel transform, and scaling operations are all the special cases of the LCT. The LCT can extend their utilities and applications and can solve some problems that cannot be solved well by these operations.

\section{B. Eigenfunctions of Fractional Fourier Transform \\ In [7], Namias discussed that the FRFT has the eigenfunctions} as

$$
\phi_{m}(t)=\frac{1}{\sqrt{2^{m} m ! \sqrt{\pi}}} \cdot e^{-t^{2} / 2} \cdot H_{m}(t) \quad m \in[0,1,2,3, \ldots]
$$

where $H_{m}(t)$ is the Hermite function of order $m$ :

$$
H_{m}(t)=(-1)^{m} \cdot e^{t^{2}} \cdot \frac{d^{m}}{d t^{m}}\left(e^{-t^{2}}\right)
$$

and the corresponding eigenvalue for $\phi_{m}(t)$ is $\exp (-j m \alpha)$, i.e.,

$$
O_{F}^{\alpha}\left(\phi_{m}(t)\right)=e^{-j \cdot m \cdot \alpha} \cdot \phi_{m}(t) .
$$

The eigenfunctions of FRFT satisfy the orthogonality property

$$
\int_{-\infty}^{\infty} \phi_{m}(t) \cdot \phi_{n}(t) \cdot d t=\delta_{m, n}
$$

Maybe (17) is the only set of eigenfunctions for the FRFT when $\alpha / 2 \pi$ is not a rational number. We can say that at least no eigenfunctions other than (17) have been found when $\alpha / 2 \pi$ is not a rational number until now, but when $\alpha / 2 \pi$ is a rational number, the FRFT has the eigenfunctions other than (17). For example, when $\alpha=0$ (in this case, the FRFT becomes the identity operation), all functions will be the eigenfunctions of the FRFT. When $\alpha=\pi$ (in this case, the FRFT becomes the time inverse operation), all even and odd functions will be the eigenfunctions 
of the FRFT, and when $\alpha= \pm \pi / 2$ (in this case, the FRFT becomes the forward and inverse FT), the following functions are all the eigenfunctions of the FRFT (see [20, ch. 22 ])

$$
\begin{aligned}
& \text { 1) } \sum_{p=-\infty}^{\infty} \delta(x-p \sqrt{2 \pi}) \\
& \text { 2) } \sin \left(\sqrt{\frac{\pi}{2}} x\right) \sum_{p=-\infty}^{\infty} \delta(x-(p+0.5) \sqrt{2 \pi}) \\
& \text { 3) }\left|\frac{x}{\sqrt{2 \pi}}\right|^{(-1 / 2)} \text { 4) }\left|\frac{x}{\sqrt{2 \pi}}\right|^{-1 / 2} \operatorname{sgn}(x) \\
& \text { 5) } \operatorname{sech}\left(\sqrt{\frac{\pi}{2}} \cdot x\right) .
\end{aligned}
$$

Recently, in many papers (such as [21] and [22]), it has been discussed, for the case where $\alpha=2 \pi N / M$, where $N$ and $M$ are integers, that the FRFT also has eigenfunctions other than (17).

The eigenfunctions of the FRFT (which is also called as the self-fractional Fourier functions) are useful for the analysis of optical system and wave propagation, especially for the analysis of self-imaging phenomena [17] and resonance phenomena [23].

We have shown that the FRFT is just the LCT with parameters $\{\cos \alpha, \sin \alpha,-\sin \alpha, \cos \alpha\} \quad$ multiplied by $\left(e^{j \alpha}\right)^{1 / 2}$ [see (10)]. Therefore, the LCT with parameters $\{\cos \alpha, \sin \alpha,-\sin \alpha, \cos \alpha\}$ also has the eigenfunctions set as (17), but the eigenvalues are modified as $\left(e^{-j \alpha}\right)^{1 / 2} \cdot \exp (-j m \alpha)$ :

$$
\begin{aligned}
O_{F}^{(\cos \alpha, \sin \alpha,-\sin \alpha, \cos \alpha)}\left(\phi_{m}(t)\right) & \\
& =\left(e^{-j \alpha}\right)^{1 / 2} e^{-j \cdot m \cdot \alpha} \cdot \phi_{m}(t) .
\end{aligned}
$$

\section{Previous Study on the Eigenfunctions of the LCT}

Recently, in [12], they have found the eigenfunctions of the linear canonical transform (the LCT) with the parameters $\{a, b, c, d\}$ as

$$
\begin{aligned}
\phi_{m}^{(\sigma, \tau)}(t) & =\frac{1}{\sqrt{\sigma \cdot 2^{m} m ! \sqrt{\pi}}} \exp \left(-\frac{(1+i \tau) t^{2}}{2 \sigma^{2}}\right) \cdot H_{m}\left(\frac{t}{\sigma}\right) \\
m & =0,1,2,3 \ldots
\end{aligned}
$$

where $H_{m}(t)$ is Hermite function [see (18)], and the corresponding eigenvalues are

$\lambda_{m}=\exp \left(-i \alpha m+\varepsilon_{\alpha}\right) \varepsilon_{\alpha}$ is some constant dependent on $\alpha$

and the values of $\sigma, \tau$, and $\alpha$ are

$$
\begin{aligned}
\sigma^{2} & =\frac{2 b}{\sqrt{4-(a+d)^{2}}}, \quad \tau=\frac{a-d}{\sqrt{4-(a+d)^{2}}} \\
\alpha & =\cos ^{-1}\left(\frac{a+d}{2}\right) .
\end{aligned}
$$

In converse, the original parameters $\{a, b, c, d\}$ can be represented by $\{\sigma, \tau, \alpha\}$ as

$$
\begin{aligned}
& a=\cos \alpha+\tau \sin \alpha, \quad b=\sigma^{2} \sin \alpha \\
& c=-\left(\tau^{2}+1\right) \cdot \frac{\sin \alpha}{\sigma^{2}}, \quad d=\cos \alpha-\tau \sin \alpha .
\end{aligned}
$$

Then, the following equality will be satisfied:

$$
a+d=a_{2}+d_{2} .
$$

This property can be proved by direct calculation or by the theorem that the eigenvalues of two similar matrices are the same, and the diagonal sum of a matrix is its eigenvalues sum [24].

Property $\quad B$ : Suppose $\quad\{a, b, c, d\}, \quad\left\{a_{1}, b_{1}, c_{1}, d_{1}\right\}$, and $\left\{a_{2}, b_{2}, c_{2}, d_{2}\right\}$ have the relation as (27), and $a d-b c=a_{1} d_{1}-b_{1} c_{1}=a_{2} d_{2}-b_{2} c_{2}=1$; then, the LCT with parameters $\{a, b, c, d\}$ can be decomposed as

$$
\begin{aligned}
& O_{F}^{(a, b, c, d)}(f(t))=O_{F}^{\left(a_{1}, b_{1}, c_{1}, d_{1}\right)} \\
& \cdot\left(O_{F}^{\left(a_{2}, b_{2}, c_{2}, d_{2}\right)}\left(O_{F}^{\left(d_{1},-b_{1},-c_{1}, a_{1}\right)}(f(t))\right)\right) .
\end{aligned}
$$


If we know that $e(t)$ is the eigenfunction of the LCT with parameters $\left\{a_{2}, b_{2}, c_{2}, d_{2}\right\}$ and the corresponding eigenvalue is $\lambda$

$$
O_{F}^{\left(a_{2}, b_{2}, c_{2}, d_{2}\right)}(e(t))=\lambda \cdot e(t)
$$

then $O_{F}^{\left(a_{1}, b_{1}, c_{1}, d_{1}\right)}(e(t))$ will be the eigenfunction of the LCT with parameters $\{a, b, c, d\}$, and the corresponding eigenvalue is also $\lambda$ :

$$
\begin{aligned}
O_{F}^{(a, b, c, d)}\left(O_{F}^{\left(a_{1}, b_{1}, c_{1}, d_{1}\right)}(e(t))\right) \\
=O_{F}^{\left(a_{1}, b_{1}, c_{1}, d_{1}\right)} \\
\quad \cdot\left(O _ { F } ^ { ( a _ { 2 } , b _ { 2 } , c _ { 2 } , d _ { 2 } ) } \left(O_{F}^{\left(d_{1},-b_{1},-c_{1}, a_{1}\right)}\right.\right. \\
\left.\left.\quad \cdot\left(O_{F}^{\left(a_{1}, b_{1}, c_{1}, d_{1}\right)}(e(t))\right)\right)\right) \\
=O_{F}^{\left(a_{1}, b_{1}, c_{1}, d_{1}\right)}\left(O_{F}^{\left(a_{2}, b_{2}, c_{2}, d_{2}\right)}(e(t))\right) \\
=\lambda \cdot O_{F}^{\left(a_{1}, b_{1}, c_{1}, d_{1}\right)}(e(t)) .
\end{aligned}
$$

\section{B. Eigenfunctions of the LCT for the Cases Where $|a+d|<2$}

Here, we use Properties A and B introduced in Section III-A to derive the eigenfunctions. Since $-2<2 \cos \alpha<2$, from Property A, we conclude that when $|a+d|<2$, the $a b c d$ matrix of the LCT can be decomposed as

$$
\begin{aligned}
{\left[\begin{array}{ll}
a & b \\
c & d
\end{array}\right] } & =\left[\begin{array}{ll}
a_{1} & b_{1} \\
c_{1} & d_{1}
\end{array}\right]\left[\begin{array}{cc}
\cos \alpha & \sin \alpha \\
-\sin \alpha & \cos \alpha
\end{array}\right]\left[\begin{array}{ll}
a_{1} & b_{1} \\
c_{1} & d_{1}
\end{array}\right]^{-1} \\
& =\left[\begin{array}{ll}
a_{1} & b_{1} \\
c_{1} & d_{1}
\end{array}\right]\left[\begin{array}{cc}
\cos \alpha & \sin \alpha \\
-\sin \alpha & \cos \alpha
\end{array}\right]\left[\begin{array}{cc}
d_{1} & -b_{1} \\
-c_{1} & a_{1}
\end{array}\right]
\end{aligned}
$$

where $a_{1} d_{1}-b_{1} c_{1}=1$. Because, from Property A, $a+d=$ $\cos \alpha+\cos \alpha$, we have

$$
\alpha=\cos ^{-1}\left(\frac{a+d}{2}\right) .
$$

Therefore, when $|a+d|<2$, the LCT can be decomposed as $O_{F}^{(a, b, c, d)}(g(t))=O_{F}^{\left(a_{1}, b_{1}, c_{1}, d_{1}\right)}$

$$
\text { - }\left(O_{F}^{(\cos \alpha, \sin \alpha,-\sin \alpha, \cos \alpha)}\left(O_{F}^{\left(d_{1},-b_{1},-c_{1}, a_{1}\right)}(g(t))\right)\right)
$$

i.e., decomposed as the combination of the FRFT and the LCTs with parameters $\left\{a_{1}, b_{1}, c_{1}, d_{1}\right\}$ and $\left\{d_{1},-b_{1},-c_{1}, a_{1}\right\}$. Then, from Property B, the LCT will have the eigenfunctions set as

$$
\left\{O_{F}^{\left(a_{1}, b_{1}, c_{1}, d_{1}\right)}\left(\phi_{m}(t)\right), \quad m=0,1,2, \ldots\right\}
$$

where $\phi_{m}(t)$ is the eigenfunctions of the FRFT defined as (17).

There are many possible choices for $\left\{a_{1}, b_{1}, c_{1}, d_{1}\right\}$ in (32)-(35). We can choose them as

$$
\left[\begin{array}{ll}
a_{1} & b_{1} \\
c_{1} & d_{1}
\end{array}\right]=\left[\begin{array}{cc}
\sigma & 0 \\
-\tau \sigma^{-1} & \sigma^{-1}
\end{array}\right] \text {. }
$$

In addition, the LCT with these parameters is the combination of scaling and chirp multiplication

$$
O_{F}^{\left(a_{1}, b_{1}, c_{1}, d_{1}\right)}(g(t))=\sigma^{-1 / 2} \cdot e^{-(j / 2) \cdot\left(\tau / \sigma^{2}\right) \cdot t^{2}} \cdot g\left(\sigma^{-1} \cdot t\right) .
$$

Then, from (17) and (37), we conclude that when $|\boldsymbol{a}+\boldsymbol{d}|<\mathbf{2}$, the LCT will have the eigenfunctions

$$
\begin{aligned}
\phi_{m}^{(\sigma, \tau)}(t) & =O_{F}^{\left(a_{1}, b_{1}, c_{1}, d_{1}\right)}\left(\phi_{m}(t)\right) \\
& =\sigma^{-1 / 2} \cdot e^{(j / 2) \cdot \tau \cdot t^{2}} \cdot \phi_{m}\left(\frac{t}{\sigma}\right)
\end{aligned}
$$

$$
\begin{aligned}
& \text { i.e., } \\
& \begin{aligned}
& \phi_{m}^{(\sigma, \tau)}(t)=\frac{1}{\sqrt{\sigma \cdot 2^{m} m ! \sqrt{\pi}}} \\
& \cdot \exp \left(\frac{-(1+i \tau) t^{2}}{2 \sigma^{2}}\right) \cdot H_{m}\left(\frac{t}{\sigma}\right)
\end{aligned}
\end{aligned}
$$

where $H_{m}(t)$ is Hermite function [see (18)], and the corresponding eigenvalues are the same as the eigenvalues of the LCT with parameters $\{\cos \alpha, \sin \alpha,-\sin \alpha, \cos \alpha\}$ [see (22)]

$$
\lambda_{m}=\left(e^{-j \alpha}\right)^{1 / 2} \cdot \exp (-i \alpha m) \quad(\alpha \text { is defined as }(33)) \text {. }
$$

Since, after substituting (36) into (32), we obtain

$$
\begin{aligned}
{\left[\begin{array}{ll}
a & b \\
c & d
\end{array}\right] } & =\left[\begin{array}{cc}
\sigma & 0 \\
-\tau \sigma^{-1} & \sigma^{-1}
\end{array}\right]\left[\begin{array}{cc}
\cos \alpha & \sin \alpha \\
-\sin \alpha & \cos \alpha
\end{array}\right]\left[\begin{array}{cc}
\sigma^{-1} & 0 \\
\tau \sigma^{-1} & \sigma
\end{array}\right] \\
& =\left[\begin{array}{cc}
\cos \alpha+\tau \sin \alpha & \sigma^{2} \cdot \sin \alpha \\
-\left(\tau^{2}+1\right) \frac{\sin \alpha}{\sigma^{2}} & \cos \alpha-\tau \sin \alpha
\end{array}\right]
\end{aligned}
$$

from (41), we see that the explicit values of $\sigma, \tau$, and $\alpha$ are

$$
\begin{aligned}
\sigma^{2} & =\frac{2|b|}{\sqrt{4-(a+d)^{2}}}, \quad \tau=\frac{\operatorname{sgn}(b) \cdot(a-d)}{\sqrt{4-(a+d)^{2}}}, \\
\alpha & =\cos ^{-1}\left(\frac{a+d}{2}\right)=\sin ^{-1}\left(\frac{\operatorname{sgn}(b)}{2} \sqrt{4-(a+d)^{2}}\right) .
\end{aligned}
$$

Equations (39), (40), and (42) are the eigenfunctions and eigenvalues we solved for the LCT in the case where $|a+d|<2$. This result is more complete than the result in [12] [see (23)-(25)].

We can show, as in the case of the FRFT, the eigenfunctions of the LCT when $|a+d|<2$ have the orthogonality property

$\int_{-\infty}^{\infty} \phi_{m}^{(\sigma, \tau)}(t) \cdot \bar{\phi}_{n}^{(\sigma, \tau)}(t) \cdot d t=\delta_{m, n}$

(upper bar means conjugation).

We may ask, except for (39), whether the LCT has other eigenfunctions when $|a+d|<2$. We discuss it below.

From Properties A and B, since $a+d=a_{2}+d_{2}$ and the eigenfunctions of the LCT with parameters $\left\{a_{2}, b_{2}, c_{2}, d_{2}\right\}$ should have been known, the only possible choice for $\left\{a_{2}, b_{2}, c_{2}, d_{2}\right\}$ when $|a+d|<2$ is $\{\cos \alpha, \sin \alpha,-\sin \alpha, \cos \alpha\}$, and $\alpha$ should satisfy (42). Thus, to find other eigenfunctions of the LCT for the case where $|a+d|<2$, we must try to find the values of $\left\{a_{1}, b_{1}, c_{1}, d_{1}\right\}$ other than (36) that can satisfy (32). Because for any values of $\left\{a_{1}, b_{1}, c_{1}, d_{1}\right\}$ where $a_{1} d_{1}-b_{1} c_{1}=1$, we can decompose its $2 \times 2$ matrix as

$$
\begin{aligned}
{\left[\begin{array}{ll}
a_{1} & b_{1} \\
c_{1} & d_{1}
\end{array}\right] } & =\left[\begin{array}{cc}
\sigma & 0 \\
-\tau \sigma^{-1} & \sigma^{-1}
\end{array}\right] \cdot\left[\begin{array}{ll}
a_{3} & b_{3} \\
c_{3} & d_{3}
\end{array}\right] \\
\left(a_{3} d_{3}-b_{3} c_{3}\right. & =1)
\end{aligned}
$$

where $\tau, \sigma$ satisfies (42). Then, from (32), and after some calculation, we can prove that the values of $\left\{a_{3}, b_{3}, c_{3}, d_{3}\right\}$ must be of the following form:

$$
a_{3}=\cos \beta, \quad b_{3}=\sin \beta, \quad c_{3}=-\sin \beta, \quad d_{3}=\cos \beta
$$

where $\beta$ is any real number. Thus

$$
\left[\begin{array}{ll}
a_{1} & b_{1} \\
c_{1} & d_{1}
\end{array}\right]=\left[\begin{array}{cc}
\sigma & 0 \\
-\tau \sigma^{-1} & \sigma^{-1}
\end{array}\right] \cdot\left[\begin{array}{cc}
\cos \beta & \sin \beta \\
-\sin \beta & \cos \beta
\end{array}\right] .
$$


Then, from Property B and the values of $\left\{a_{1}, b_{1}, c_{1}, d_{1}\right\}$ as above, we conclude that the LCT will have the eigenfunctions as $\left(\phi_{m}(t)\right.$ is the eigenfunction of FRFT)

$$
\begin{aligned}
O_{F}^{\left(a_{1}, b_{1}, c_{1}, d_{1}\right)}\left(\phi_{m}(t)\right)= & O_{F}^{\left(\sigma, 0,-\tau \sigma^{-1}, \sigma^{-1}\right)} \\
& \cdot\left(O_{F}^{(\cos \beta, \sin \beta,-\sin \beta, \cos \beta)}\left(\phi_{m}(t)\right)\right) \\
= & O_{F}^{\left(\sigma, 0,-\tau \sigma^{-1}, \sigma^{-1}\right)} \\
& \cdot\left(\left(e^{-j a}\right)^{1 / 2} e^{-j m \beta} \phi_{m}(t)\right) \\
= & \sqrt{\sigma} \cdot\left(e^{-j \alpha}\right)^{1 / 2} e^{(j / 2) \tau \cdot t^{2}-j m \beta} \\
& \cdot \phi_{m}(\sigma \cdot t) \\
O_{F}^{\left(a_{1}, b_{1}, c_{1}, d_{1}\right)}\left(\phi_{m}(t)\right)= & \sqrt{\sigma} \cdot\left(e^{-j \alpha}\right)^{1 / 2} \\
& \cdot \exp \left(-\frac{(1+i \tau) t^{2}}{2 \sigma^{2}}-j m \beta\right) \\
& \cdot H_{m}\left(\frac{t}{\sigma}\right) .
\end{aligned}
$$

In fact, except for some difference of constant phase, (48) is all then the same as (39). No new eigenfunction has been found.

Thus, in most of the cases, (39) are the only possible eigenfunctions of the LCT when $|a+d|<2$, but in the case where $\alpha / 2 \pi$ [ $\alpha$ is defined as (33)] is a rational number

$$
\alpha=\cos ^{-1}\left(\frac{a+d}{2}\right)=2 \pi \frac{N}{M}, \text { where } M, N \text { are integers }
$$

we can also find other eigenfunctions of the LCT. In fact, if (49) is satisfied, then

$\sum_{k=0}^{\infty} c_{k} \cdot \phi_{s+N k}^{(\sigma, \tau)}(t) \quad$ where $\phi_{m}^{(\sigma, \tau)}(t)$ is defined as (39)

$c_{k}$ 's are any constants

will also be the eigenfunctions of the LCT when $|a+d|<2$, and the corresponding eigenvalue is $\left(e^{-j \alpha}\right)^{-(1 / 2)} \cdot \exp (-j \alpha \cdot s)$ (see [25]).

\section{EIGENFUnCtions OF THE LCT WhEN $|a+d|=2$}

The eigenfunctions of the LCT derived in Section III is only suitable for the case where $|a+d|<2$. For the cases where $|a+d|=2$ or $|a+d|>2$, we cannot apply the results in Section III. In this section, we discuss the eigenfunctions of the LCT when $|a+d|=2$.

We first discuss the case where $a+d= \pm 2$ and $b=0$ because these are the simplest cases. Then, we use these results and the properties introduced in Section III-A to derive the eigenfunctions for the cases where $\{a, b, c, d\}=\{1, b, 0,1\}$ and $\{-1, b, 0,-1\}$. In these cases, the LCT becomes the Fresnel transform and the Fresnel transform combined with reverse operation. We find, except for periodic functions, that the almostperiodic functions are also the eigenfunctions of the Fresnel transform. The conventional theory of Talbot effects can be generalized by our results. Then, we will use the eigenfunctions of the Fresnel transform and the Fresnel transform combined with reverse operation to discuss the eigenfunctions of the LCT for all the other cases where $|a+d|=2$.

\section{A. Case Where $a+d=2$ and $b=0$}

Since $a d-b c=1$, for the case where $a+d=2$ and $b=0$, the parameters $\{a, b, c, d\}$ of the LCT must be of the form

$$
\left[\begin{array}{ll}
a & b \\
c & d
\end{array}\right]=\left[\begin{array}{ll}
1 & 0 \\
c & 1
\end{array}\right]
$$

In this case, therefore, the LCT becomes the chirp multiplication operation

$$
O_{F}^{(1,0, c, 1)}(f(t))=e^{j \cdot c \cdot u^{2} / 2} \cdot f(u) .
$$

The eigenfunctions of any multiplication operation are the form of impulse train

$\varphi(t)=\sqrt{E^{-1}} \sum_{n=-\infty}^{\infty} A_{n} \cdot \delta\left(t-s_{n}\right)$, where $E=\sum_{n=-\infty}^{\infty}\left|A_{n}\right|^{2}$.

We can show that if $s_{n}$ s satisfy

$$
\begin{aligned}
\cdots & =e^{(j / 2) c \cdot s_{-1}^{2}}=e^{(j / 2) \cdot c \cdot s_{0}^{2}}=e^{(j / 2) \cdot c \cdot s_{1}^{2}} \\
& =e^{(j / 2) \cdot c \cdot s_{2}^{2}}=\cdots
\end{aligned}
$$

$$
O_{F}^{(1,0, c, 1)}(\varphi(t))=e^{j \cdot c \cdot s_{0}^{2} / 2} \cdot \varphi(u) .
$$

Therefore, we conclude that the eigenfunction of the LCT when $a+d=2$ and $b=0$ must be of the form

$$
\begin{aligned}
\phi_{B}^{c, h}(t)=\sqrt{E^{-1}} \cdot & {\left[\sum_{n=0}^{\infty} A_{n} \cdot \delta\left(t-\sqrt{\frac{4 n \pi}{|c|}+h}\right)\right.} \\
& \left.+\sum_{m=0}^{\infty} B_{m} \cdot \delta\left(t+\sqrt{\frac{4 m \pi}{|c|}+h}\right)\right]
\end{aligned}
$$

where $0 \leq h<4 \pi /|c|, A_{n} \mathrm{~s}$, ans $B_{m} \mathrm{~s}$ are free to choose

$$
E=\sum_{n=0}^{\infty}\left(\left|A_{n}\right|^{2}+\left|B_{n}\right|^{2}\right)
$$

and the corresponding eigenvalue is

$$
\lambda_{c, h}=\exp \left(\frac{j c h}{2}\right) .
$$

B. Case Where $a+d=-2$ and $b=0$

For this case, we can prove that the parameters $\{a, b, c, d\}$ must be of the following form:

$$
\left[\begin{array}{ll}
a & b \\
c & d
\end{array}\right]=\left[\begin{array}{cc}
-1 & 0 \\
c & -1
\end{array}\right]
$$

In addition, the formula of the LCT in this case becomes

$$
O_{F}^{(1,0, c, 1)}(f(t))=(-1)^{1 / 2} \cdot e^{-j \cdot c \cdot u^{2} / 2} \cdot f(-u) .
$$

This is the combination of chirp multiplication and time reverse operation. Therefore, the eigenfunctions in this case are the form of the symmetric or anti-symmetric impulse trains

$$
\begin{aligned}
& \phi_{C}^{c, h}(t)= \sqrt{E^{-1}} \cdot \sum_{n=0}^{\infty} A_{n} \\
& \cdot\left\{\delta\left(t-\sqrt{4 n \pi|c|^{-1}+h}\right)\right. \\
&\left.+\delta\left(t+\sqrt{4 n \pi|c|^{-1}+h}\right)\right\}
\end{aligned}
$$




$$
\text { or } \begin{aligned}
\phi_{C}^{c, h}(t)= & \sqrt{E^{-1}} \cdot \sum_{n=0}^{\infty} A_{n} \\
& \cdot\left\{\delta\left(t-\sqrt{4 n \pi|c|^{-1}+h}\right)\right. \\
& \left.\quad-\delta\left(t+\sqrt{4 n \pi|c|^{-1}+h}\right)\right\}
\end{aligned}
$$

where $0 \leq h<4 \pi /|c|, A_{n}$ s are free to choose

$$
E=2 \sum_{n=0}^{\infty}\left|A_{n}\right|^{2}
$$

and the corresponding eigenvalues are

$$
\begin{aligned}
& \lambda_{c, h}=(-1)^{1 / 2} \exp \left(\frac{j c h}{2}\right) \text { for }(61) \\
& \lambda_{c, h}=-(-1)^{1 / 2} \exp \left(\frac{j c h}{2}\right) \text { for }(62) .
\end{aligned}
$$

C. Eigenfunctions of the LCT When $\{a, b, c, d\}=$ $\{ \pm 1, b, 0, \pm 1\}$

We know that the LCT with parameters $\{a, b, c, d\}$ is just the 1-D Fresnel transform [see (14) and (15)]. The Fresnel transform describes the monochromic light propagation through free space. From the theory of the Talbot effect [16], [17], if the distribution of input light $f(x, 0)$ is periodic, i.e., $f(x, 0)=$ $f(x+q, 0)$, then after propagation through free space, the intensity of the light at the distance $N \cdot z$ will be the same as the original, i.e.,

$$
\begin{aligned}
|f(x, N \cdot z)| & =|f(x, 0)|, \\
\text { where } z & =\frac{2 q^{2}}{\lambda} \text { (Talbot distance), } N \text { is an integer. }
\end{aligned}
$$

Thus, together with (14) and (15), we can conclude that if $e(t)$ is periodic and has the period of $q$, then it is the eigenfunctions of the LCT with parameters $\left\{1, N q^{2} / \pi, 0,1\right\}$, where $N$ is some integer

$$
O_{F}^{\left(1, S q^{2} / \pi, 0,1\right)}(e(t))=\tau \cdot e(t) \text { if } e(t)=e(t+q) .
$$

Besides, we can prove the corresponding eigenvalue is just 1 , i.e., $\tau=1$. The above equation can also be rewritten as

$$
\begin{aligned}
& O_{F}^{(1, b, 0,1)}(e(t))=e(t) \\
& \text { if } e(t)=e\left(t+\sqrt{\frac{|b| \pi}{N}}\right)
\end{aligned}
$$

( $N$ is some positive integer).

The above result comes from the Talbot effect. It can be generalized. Suppose $g(t)=g(t+q)$ and that $g_{o}(v)$ is the LCT of $g(t)$ with parameters $\left\{1, q^{2} N / \pi M, 0,1\right\}$, i.e., the periodic monochromic light propagating through the distance of $z_{\mathrm{T}} N / M$, where $z_{\mathrm{T}}$ is the Talbot distance, then [26]:

$$
\begin{aligned}
g_{o}(v)= & O_{F}^{\left(1,\left(q^{2} N / \pi M\right), 0,1\right)}(g(t)) \\
= & \frac{1}{M} \sum_{p=0}^{M-1} g\left(v-\frac{p q}{M}\right) \\
& \cdot \sum_{n=0}^{M-1} e^{j(2 \pi / M)\left(p n-N n^{2}\right)} .
\end{aligned}
$$

That is, $g_{o}(v)$ is the linear combination of $g(v-p q / M)$. This is called as the fractional Talbot effect[26]-[28]. The original Talbot effect is the special case where $M=N=1$.

From (68), we can generalize the results in (66) and (67). Suppose $\left[1, a_{1}, a_{2}, \ldots, a_{M-1}\right]^{\mathrm{T}}$ is the eigenvector of the following matrix:

$$
\begin{gathered}
{\left[\begin{array}{ccccc}
c_{0} & c_{M-1} & c_{M-2} & \cdots & c_{1} \\
c_{1} & c_{0} & c_{M-1} & \cdots & c_{2} \\
c_{2} & c_{1} & c_{0} & \cdots & c_{3} \\
\vdots & \vdots & \vdots & \ddots & \vdots \\
c_{M-1} & c_{M-2} & c_{M-3} & \cdots & c_{0}
\end{array}\right]} \\
\text { where } c_{p}=\frac{1}{M} \sum_{n=0}^{M-1} e^{j(2 \pi / M)\left(p n-N n^{2}\right)}
\end{gathered}
$$

and its corresponding eigenvalue is $\lambda$. If $g(x)=g(x+q)$, and

$$
\begin{aligned}
g(x): g\left(x+\frac{q}{M}\right) & : g\left(x+\frac{2 q}{M}\right): \ldots: g\left(x+\frac{(M-1) q}{M}\right) \\
& =1: a_{1}: a_{2}: \ldots: a_{M-1} \text { for } x \in\left(0, \frac{q}{M}\right)
\end{aligned}
$$

then we can show that $g(x)$ is the eigenfunction of the LCT with parameters $\left\{1, N q^{2} / \pi M, 0,1\right\}$, and the corresponding eigenvalues are also $\lambda$. Although there is no simple expression for the eigenvectors of the matrix in (69), its eigenvalues can be expressed in the closed form as

$$
\lambda_{k}=\exp \left(\frac{-j 2 \pi N k^{2}}{M}\right) \text { where } k=0,1,2, \ldots, M-1 .
$$

In (67), we find that the function with period of $q=\sqrt{|b| \pi / N}$ is the eigenfunction of the LCT with parameters $\{1, b, 0,1\}$. Here, we also show that the function with period $q=\sqrt{|\eta| \pi M / N}$ is also the eigenfunction of the LCT with parameters $\{1, b, 0,1\}$ if it satisfies some symmetry relation.

In fact, except for periodic eigenfunctions, the LCT with parameters $\{1, b, 0,1\}$ and, hence, the Fresnel transform, also have some aperiodic eigenfunctions.

We can apply the eigenfunctions of the LCT with parameters $\{1,0, c, 1\}$ derived in Section IV-A together with Properties A and B introduced in Section III-A to derive the eigenfunctions of the LCT with parameters $\{1, b, 0,1\}$. Since

$$
\left[\begin{array}{ll}
1 & b \\
0 & 1
\end{array}\right]=\left[\begin{array}{cc}
0 & 1 \\
-1 & 0
\end{array}\right] \cdot\left[\begin{array}{cc}
1 & 0 \\
-b & 1
\end{array}\right] \cdot\left[\begin{array}{cc}
0 & -1 \\
1 & 0
\end{array}\right] .
$$

Therefore, from Property B, if we do the Fourier transform for the eigenfunctions of the LCT with parameters $\{1,0,-b, 1\}$, then it is just the eigenfunctions of the LCT with parameters $\{1, b, 0,1\}$. Therefore, from (56), the LCT with parameters $\{1, b, 0,1\}$ will have the eigenfunctions as

$$
\begin{aligned}
\psi^{b, h}(t)= & \mathrm{FT}\left(\phi_{B}^{-b, h}(t)\right) \\
= & \sqrt{\frac{1}{2 \pi E}} \cdot\left[\sum_{n=0}^{\infty} A_{n} \cdot \exp \left(j \cdot t \sqrt{\frac{4 n \pi}{|b|}+h}\right)\right. \\
& \left.+\sum_{m=0}^{\infty} B_{m} \cdot \exp \left(-j \cdot t \sqrt{\frac{4 m \pi}{|b|}+h}\right)\right]
\end{aligned}
$$


where $0 \leq h<4 \pi /|b|, A_{n}$ s, and $B_{m}$ s are free to choose

$$
E=\sum_{n=0}^{\infty}\left(\left|A_{n}\right|^{2}+\left|B_{n}\right|^{2}\right)
$$

and the corresponding eigenvalues are the same as the eigenvalues of the LCT with parameters $\{1,0,-b, 1\}$ [see (58)]

$$
\lambda_{b, h}=\exp \left(-\frac{j b h}{2}\right) .
$$

Equation (75) is the general form of the eigenfunctions of the LCT with parameters $\{1, b, 0,1\}$. Equation (73) is usually not periodic. It can be called the almost-periodic function.

Since the Fresnel transform equals the LCT with parameters $\{1, z \lambda / 2 \pi, 0,1\}$ multiplied by some constant phase [see (14) and (15)], from (73), the general form of the eigenfunctions of Fresnel transform is

$$
\begin{array}{r}
\psi_{\text {Fres }}^{z, h}(t)=\sqrt{\frac{1}{2 \pi E}} \cdot\left[\sum_{n=0}^{\infty} A_{n} \cdot \exp \left(j 2 \pi t \sqrt{\frac{2 n}{z \lambda}+h}\right)\right. \\
\left.+\sum_{m=0}^{\infty} B_{m} \cdot \exp \left(-j 2 \pi t \sqrt{\frac{2 m}{z \lambda}+h}\right)\right]
\end{array}
$$

where $0 \leq h<8 \pi^{2} / z \lambda, A_{n}$ s and $B_{m}$ s are free to choose, $E$ is defined the same as (74), and the corresponding eigenvalue is

$$
\lambda_{z, h}=\exp \left(\frac{j \pi \cdot z}{\lambda}-\frac{j z \lambda h}{4 \pi}\right)
$$

$$
\text { where } z \text { is the propagation distance. }
$$

From the conventional theories of the Talbot effect [16], [17] and fractional Talbot effect [26]-[28], we know that periodic functions are the eigenfunctions of the Fresnel transform, but from (76), we find that some almost-periodic functions are also the eigenfunctions of the Fresnel transform. This type of aperiodic function will also cause the self-imaging phenomena after free space propagation. The conventional Talbot effect is just the special case of (76) where

$h=0, \quad A_{n}=B_{m}=0$

$$
\text { when } n, m \neq N^{2} \text { where } N \text { is some integer. }
$$

Then, we discuss the eigenfunctions of the next LCT with parameters $\{-1, b, 0,-1\}$. Similarly, we can apply the eigenfunctions of the LCT with parameters $\{-1,0, c,-1\}$ derived in Section IV-B and Properties A and B introduced in Section III-A. Since

$$
\left[\begin{array}{cc}
-1 & b \\
0 & -1
\end{array}\right]=\left[\begin{array}{cc}
0 & 1 \\
-1 & 0
\end{array}\right] \cdot\left[\begin{array}{cc}
-1 & 0 \\
-b & -1
\end{array}\right] \cdot\left[\begin{array}{cc}
0 & -1 \\
1 & 0
\end{array}\right]
$$

the transform results of the FT for the eigenfunctions of the LCT with parameters $\{-1,0,-b,-1\}$ [see (61) and (62)] is just the eigenfunctions of the LCT with parameters $\{-1, b, 0,-1\}$

$$
\begin{aligned}
\mu^{b, h}(t)= & \mathrm{FT}\left(\phi_{C}^{-b, h}(t)\right) \\
= & \sqrt{\frac{1}{2 \pi E}} \cdot \sum_{n=0}^{\infty} A_{n} \cos \left(t \sqrt{\frac{4 n \pi}{|b|}+h}\right) \\
& {[\text { from (61)] }}
\end{aligned}
$$

$$
\text { or } \begin{aligned}
\mu^{b, h}(t) & =\mathrm{FT}\left(\phi_{C}^{-b, h}(t)\right) \\
& =\sqrt{\frac{1}{2 \pi E}} \cdot \sum_{n=0}^{\infty} A_{n} \sin \left(t \sqrt{\frac{4 n \pi}{|b|}+h}\right)
\end{aligned}
$$$$
\text { [from (62)] }
$$

where $0 \leq h<4 \pi /|b|, A_{n}$ s are free to choose

$$
E=\frac{\left[\sum_{n=0}^{\infty}\left|A_{n}\right|^{2}\right]}{2} \text {. }
$$

Equation (80) can be called the almost-periodic symmetric function, and (81) can be called the almost-periodic anti-symmetric function. The corresponding eigenvalues are

$$
\begin{aligned}
& \lambda_{b, h}=(-1)^{1 / 2} \exp \left(\frac{-j b h}{2}\right) \text { for }(80) \\
& \lambda_{c, h}=(-1)^{1 / 2} \exp \left(\frac{-j b h}{2}\right) \text { for }(81) .
\end{aligned}
$$

Since the eigenfunctions of the LCT with parameters $\{ \pm 1, b, 0, \pm 1\}$ have been derived, we can use these results to derive the eigenfunctions of the LCT for the cases that $a+d= \pm 2$ and $b \neq 0$.

\section{Case Where $a+d=2$ and $b \neq 0$}

In this case, from Property A, $a_{2}+d_{2}=2$, and when we want to apply Property B, the eigenfunctions of the LCT with parameters $\left\{a_{2}, b_{2}, c_{2}, d_{2}\right\}$ should have been known. Therefore, we set $\left\{a_{2}, b_{2}, c_{2}, d_{2}\right\}$ as $\{1, \eta, 0,1\}$ in (27). Then

$$
\begin{aligned}
{\left[\begin{array}{ll}
a & b \\
c & d
\end{array}\right] } & =\left[\begin{array}{ll}
a_{1} & b_{1} \\
c_{1} & d_{1}
\end{array}\right] \cdot\left[\begin{array}{cc}
1 & \eta \\
0 & 1
\end{array}\right] \cdot\left[\begin{array}{cc}
d_{1} & -b_{1} \\
-c_{1} & a_{1}
\end{array}\right] \\
& =\left[\begin{array}{cc}
1-a_{1} c_{1} \eta & a_{1}^{2} \eta \\
-c_{1}^{2} \eta & 1+a_{1} c_{1} \eta
\end{array}\right] .
\end{aligned}
$$

The general solution of the above equation is

$$
\begin{gathered}
a_{1}, d_{1}: \text { free to choose, but } a_{1} \neq 0 \text { must be satisfied } \\
\quad \eta=\frac{b}{a_{1}^{2}}, \quad c_{1}=\frac{d-a}{2 b} a_{1}, \quad b_{1}=\frac{2 b\left(d_{1}-a_{1}^{-1}\right)}{d-a}
\end{gathered}
$$$$
\text { when } a \neq d \text {. }
$$

When $a=d$, the values of $\{a, b, c, d\}$ must be $\{1, b, 0,1\}$. This case has already been discussed in Section IV-C.

Then, applying Property B introduced in Section III-A, we can conclude that when $a+d=2$ and $b \neq 0$, if $f(t)$ is the eigenfunction of the LCT with parameters $\left\{1, b / a_{1}^{2}, 0,1\right\}$, i.e.,

$$
O_{F}^{\left(1, b / a_{1}^{2}, 0,1\right)}(f(t))=\lambda \cdot f(t)
$$

then the LCT with parameters $\{a, b, c, d\}$ will have the eigenfunction

$$
\phi_{D}(t)=O_{F}^{\left(a_{1}, 2 b\left(d_{1}-a_{1}^{-1}\right) /(d-a),\left((d-a) a_{1} / 2 b\right), d_{1}\right)}(f(t))
$$

where $a_{1}$ and $d_{1}$ are free to choose, and the corresponding eigenvalue is also $\lambda$. From the discussions in Section IV-C, we know 
that to satisfy (86), $f(t)$ must be the form of periodic function as

$$
\begin{aligned}
f(t)=\sqrt{\frac{1}{2 \pi E}} & \cdot\left[\sum_{n=0}^{\infty} A_{n} \exp \left(j t \sqrt{\frac{4 n a_{1}^{2} \pi}{|b|}+h}\right)\right. \\
& \left.+\sum_{m=0}^{\infty} B_{m} \exp \left(-j t \sqrt{\frac{4 m a_{1}^{2} \pi}{|b|}+h}\right)\right]
\end{aligned}
$$

where $0 \leq h<4 \pi a_{1}^{2} /|b|, A_{n}$ s and $B_{m}$ s are free to choose, $E$ is defined the same as (74), and the corresponding eigenvalue in (86) is $\lambda=\exp \left(-j b h / 2 a_{1}^{2}\right)$.

The above results can be simplified. From (85), we can prove

$$
\left[\begin{array}{ll}
a_{1} & b_{1} \\
c_{1} & d_{1}
\end{array}\right]=\left[\begin{array}{cc}
1 & 0 \\
\frac{d-a}{2 b} & 1
\end{array}\right]\left[\begin{array}{cc}
1 & a_{1} b_{1} \\
0 & 1
\end{array}\right]\left[\begin{array}{cc}
a_{1} & 0 \\
0 & a_{1}^{-1}
\end{array}\right]
$$

so that (86) can be rewritten as the combination of chirp multiplication, chirp convolution, and scaling operation as in

$$
\begin{aligned}
\phi_{D}(t)=\frac{1}{\sqrt{j 2 \pi a_{1}^{2} b_{1}}} e^{j((d-a) / 4 b) t^{2}} & \cdot \int_{-\infty}^{\infty} e^{j\left((t-x)^{2} / 2 a_{1} b_{1}\right)} f\left(\frac{x}{a_{1}}\right) d x
\end{aligned}
$$

Then, we set a new function $g(t)$ and define a new parameter $\rho$ as

$$
g(t)=\frac{1}{\left|a_{1}\right|} f\left(\frac{t}{a_{1}}\right), \quad \rho=a_{1} b_{1}=\frac{2 b\left(a_{1} d_{1}-1\right)}{d-a} .
$$

After the representation as above, we can show that the parameter $a_{1}$ has fully disappeared. In addition, because $a_{1}$ and $d_{1}$ are free to choose, if we use the parameter $\rho$ instead of $a_{1}, d_{1}$, then $\rho$ is also free to choose. From the above discussion, the results in (86)-(88) can be simplified, as in the following.

If $g(t)$ is the eigenfunction of the LCT with parameters $\{1, b, 0,1\}$

$$
\begin{aligned}
g(t)=\sqrt{\frac{1}{2 \pi S}} \cdot\left[\sum_{n=0}^{\infty} C_{n} \exp \left(j t \sqrt{\frac{4 n \pi}{|b|}+h}\right)\right. \\
\left.+\sum_{m=0}^{\infty} D_{m} \exp \left(-j t \sqrt{\frac{4 m \pi}{|b|}+h}\right)\right]
\end{aligned}
$$

where $0 \leq h<4 \pi /|b|, C_{n}$ s and $D_{m}$ s are free to choose

$$
S=\sum_{n=0}^{\infty}\left(\left|C_{n}\right|^{2}+\left|D_{n}\right|^{2}\right)
$$

then the LCT with parameters $\{a, b, c, d\}$ in the case where $a+$ $d=2$ and $b \neq 0$ will have the eigenfunction as

$\phi_{D}^{(b, \rho)}(t)=\sqrt{\frac{1}{j 2 \pi \rho}} \cdot e^{j((d-a) / 4 b) t^{2}} \cdot \int_{-\infty}^{\infty} e^{j\left((t-x)^{2} / 2 \rho\right)} g(x) d x$ where $\rho$ is free to choose (but when $a=d$, we must choose $\rho=0$ ). The corresponding eigenvalue is

$$
\lambda_{b, h}=\exp \left(-\frac{j b h}{2}\right)
$$

From the above, we can conclude that when $a+d=2$ and $b \neq 0$, the eigenfunction of the LCT is the chirp multiplication, chirp convolution of an almost-periodic function [the function in the form of (92) can be called as the almost-periodic function]. Specially, we can always choose

$$
\rho=0
$$

so that from the fact that

$$
\lim _{\rho \rightarrow 0} \frac{1}{\sqrt{j 2 \pi \rho}} \exp \left(j \frac{\left(x_{1}-x_{2}\right)^{2}}{2 \rho}\right)=\delta\left(x_{1}-x_{2}\right)
$$

we can simplify the eigenfunctions of the LCT when $a+d=2$ and $b \neq 0$ as

$$
\phi_{D}^{(b, \rho)}(t)=e^{j((d-a) / 4 b) t^{2}} \cdot g(t)
$$

It is the chirp multiplication of an almost-periodic function.

E. Case Where $a+d=-2$ and $b \neq 0$

For this case, we choose $\left\{a_{2}, b_{2}, c_{2}, d_{2}\right\}$ as $\{-1, \eta, 0,-1\}$, and (27) becomes

$$
\begin{aligned}
{\left[\begin{array}{ll}
a & b \\
c & d
\end{array}\right] } & =\left[\begin{array}{ll}
a_{1} & b_{1} \\
c_{1} & d_{1}
\end{array}\right] \cdot\left[\begin{array}{cc}
-1 & \eta \\
0 & -1
\end{array}\right] \cdot\left[\begin{array}{cc}
d_{1} & -b_{1} \\
-c_{1} & a_{1}
\end{array}\right] \\
& =\left[\begin{array}{cc}
-1-a_{1} c_{1} \eta & a_{1}^{2} \eta \\
-c_{1}^{2} \eta & -1+a_{1} c_{1} \eta
\end{array}\right] .
\end{aligned}
$$

The general solutions for $\left\{a_{1}, b_{1}, c_{1}, d_{1}\right\}$ and $\eta$ are the same as (85) when $a \neq d$. When $a=d$, the values of $\{a, b, c, d\}$ must be the same form as $\{-1, b, 0,1\}$. This case has already been discussed in Section IV-C. From (99) and Property B introduced in Section III-A, we can conclude that

$$
O_{F}^{\left(-1, b / a_{1}^{2}, 0,-1\right)}(g(t))=\lambda \cdot g(t) \quad a_{1} \text { is free to choose }
$$

i.e., $g(t)$ is the eigenfunction of the LCT with parameters $\left\{-1, b / a_{1}^{2}, 0,-1\right\}$; then

$\phi_{E}(t)=O_{F}^{\left(a_{1},\left(2 b\left(d_{1}-a_{1}^{-1}\right) /(d-a)\right),\left((d-a) a_{1} / 2 b\right), d_{1}\right)}(g(t))$

$d_{1}$ is free to choose (101)

will be the eigenfunctions of the LCT when $a+d=-2$ and $b \neq 0$, and the eigenvalue is also $\lambda$.

As in the case where $a+d=2$ and $b \neq 0$, we can also simplify the above results. From the similar process to derive (92)-(95), we obtain the results as follows. If $a+d=-2$ and 
$b \neq 0$ and $g(t)$ is the eigenfunction of the LCT with parameters $\{-1, b, 0,-1\}$, i.e., $g(t)$ satisfies [from (80) and (81)]

$$
\begin{aligned}
g(t) & =\sqrt{\frac{1}{2 \pi S}} \cdot \sum_{n=0}^{\infty} C_{n} \cos \left(t \sqrt{4 n \pi|b|^{-1}+h}\right) \\
\text { or } g(t) & =\sqrt{\frac{1}{2 \pi S}} \cdot \sum_{n=0}^{\infty} C_{n} \sin \left(t \sqrt{4 n \pi|b|^{-1}+h}\right)
\end{aligned}
$$

where $0 \leq h<4 \pi /|b|$ and $C_{n}$ s are free to choose

$$
S=\frac{\sum_{n=0}^{\infty}\left|C_{n}\right|^{2}}{2}
$$

then the LCT with parameters $\{a, b, c, d\}$ will have the eigenfunction as

$\phi_{E}^{(b, \rho)}(t)=\sqrt{\frac{1}{j 2 \pi \rho}} \cdot e^{j((d-a) / 4 b) t^{2}} \int_{-\infty}^{\infty} e^{j\left((t-x)^{2} / 2 \rho\right)} g(x) d x$

where $\rho$ is free to choose, but when $a=d$, we must choose $\rho=0$. In addition, the corresponding eigenvalue is

$$
\begin{array}{ll}
\lambda_{b, h}=(-1)^{1 / 2} \exp \left(-\frac{j b h}{2}\right) & \text { when } g(t) \text { is as (102) } \\
\lambda_{b, h}=(-1)^{1 / 2} \exp \left(-\frac{j b h}{2}\right) & \text { when } g(t) \text { is as (103). }
\end{array}
$$

Equations (105)-(107) are the eigenfunctions and eigenvalues of the LCT we derive when $a+d=-2$ and $b \neq 0$. From (102), (103), and (105), we can conclude that when $a+d=-2$ and $b \neq 0$, the eigenfunctions of the LCT are the chirp multiplication, chirp convolution of symmetric (or anti-symmetric) almost-periodic functions.

To simplify (105), we can always set

$$
\rho=0 .
$$

Then, (105) can be simplified as

$$
\phi_{E}^{(b, \rho)}(t)=e^{j((d-a) / 4 b) t^{2}} \cdot g(t) .
$$

In this case, the eigenfunctions of the LCT, when $a+d=-2$ and $b \neq 0$, are simplified as the chirp multiplication of symmetric/anti-symmetric almost-periodic functions.

From (58), (64), (95), (106), and (107), we find, as in the case where $|a+d|<2$, when $|a+d|=2$, the eigenvalues of the LCT always have unity amplitude.

We also notice that when $|a+d|<2$, there is only one complete linear independent eigenfunction set for the LCT [i.e., (39)], except for the case where $\alpha=2 \pi N / M$. When $|a+d|=$ 2 , however, there are infinite complete linear independent eigenfunctions set for the LCT.

\section{EIGENFUnCtions of the LCT WhEN $|a+d|>2$}

As in the previous cases, we also use Properties A and B introduced in Section III-A to find the eigenfunctions of the LCT when $|a+d|>2$. If we want to decompose the LCT with pa- rameters $\{a, b, c, d\}$ as (29), then since $a_{2}+d_{2}=a+d$ (from Property A), in this case, the constraint that $\left|a_{2}+d_{2}\right|>2$ must be satisfied. In addition, to apply Property $\mathrm{B}$, the eigenfunctions of the LCT with parameters $\left\{a_{2}, b_{2}, c_{2}, d_{2}\right\}$ must have been known. Therefore, in (29), we choose

$$
\begin{aligned}
\left\{a_{2}, b_{2}, c_{2}, d_{2}\right\} & =\left\{\sigma^{-1}, 0,0, \sigma\right\} \\
(\sigma & >0) \quad \text { when } a+d>2 \\
\left\{a_{2}, b_{2}, c_{2}, d_{2}\right\} & =\left\{-\sigma^{-1}, 0,0,-\sigma\right\} \\
(\sigma & >0) \quad \text { when } a+d<2 .
\end{aligned}
$$

Before discussing the eigenfunctions of the LCT when $\mid a+$ $d \mid>2$, we first discuss the eigenfunctions of the LCT with parameters $\left\{\sigma^{-1}, 0,0, \sigma\right\}$ and $\left\{-\sigma^{-1}, 0,0,-\sigma\right\}$.

\section{A. Eigenfunctions of the LCT When $\{a, b, c, d\}=$} $\left\{ \pm \sigma^{-1}, 0,0, \pm \sigma\right\}$

The LCT with parameters $\left\{\sigma^{-1}, 0,0, \sigma\right\}$ is scaling operation, and the LCT with parameters $\left\{-\sigma^{-1}, 0,0,-\sigma\right\}$ is the combination of scaling and time reverse operation

$$
\begin{aligned}
O_{F}^{\left(\sigma^{-1}, 0,0, \sigma\right)}(f(t)) & =\sigma^{1 / 2} \cdot f(\sigma \cdot u) \\
O_{F}^{\left(-\sigma^{-1}, 0,0,-\sigma\right)}(f(t)) & =(-\sigma)^{1 / 2} \cdot f(-\sigma \cdot u) \\
& =(-1)^{1 / 2} \cdot O_{F}^{\left(\sigma^{-1}, 0,0, \sigma\right)}(f(-t)) .
\end{aligned}
$$

Recently, because of the development of the fractal theory, many functions with the scaling invariance property as below have been found:

$$
f(\sigma \cdot t)=\lambda \cdot f(t)
$$

These scaling-invariant functions are also called the self-similar functions [18], [19] or fractals. In fact, the functions that satisfy the scaling invariant property of (114) will also be the eigenfunctions of the LCT with parameters $\left\{\sigma^{-1}, 0,0, \sigma\right\}$.

The simplest scaling invariant functions are the constant function, step function, impulse function, and the power of $t$

$$
\begin{aligned}
& \text { (a) constant: } O_{F}^{\left(\sigma^{-1}, 0,0, \sigma\right)}(C)=\sqrt{\sigma} \cdot C \\
& \text { (b) } s(t): O_{F}^{\left(\sigma^{-1}, 0,0, \sigma\right)}(s(t))=\sqrt{\sigma} \cdot s(u) \\
& (s(t)=1 \text { for } t \geq 0, s(t)=0 \text { for } t<0) \\
& \text { (c) } \delta(t): O_{F}^{\left(\sigma^{-1}, 0,0, \sigma\right)}(\delta(t))=\sqrt{\sigma^{-1}} \cdot \delta(u) \\
& \text { (d) } t^{n}: O_{F}^{\left(\sigma^{-1}, 0,0, \sigma\right)}\left(t^{n}\right)=\sigma^{(1 / 2)+n} \cdot u^{n} .
\end{aligned}
$$

There are also many other scaling-invariant functions developed by the fractal theory. They are all the eigenfunctions of the LCT with parameters $\left\{\sigma^{-1}, 0,0, \sigma\right\}$.

Then, we discuss the eigenfunctions of the LCT with parameters $\left\{-\sigma^{-1}, 0,0,-\sigma\right\}$. The functions satisfy the following two constraints will be the eigenfunctions of this case:
(a) $f(\sigma \cdot t)=\lambda \cdot f(t)$,
(b) $f(t)= \pm f(-t)$. 
From (119), we can conclude that the eigenfunctions of the LCT with parameters $\left\{-\sigma^{-1}, 0,0,-\sigma\right\}$ are symmetric (or anti-symmetric) scaling-invariant functions. For example, the constant function, delta function, and $t^{n}$ listed in (115), (117), and (118) all satisfy the above two constraints. They are all the eigenfunctions of the LCT with parameters $\left\{-\sigma^{-1}, 0,0,-\sigma\right\}$, but the corresponding eigenvalues are changed into $\sqrt{-\sigma}, \sqrt{-\sigma^{-1}}$, and $(-\sigma)^{n} \sqrt{-\sigma}$. Although there are also some functions that do not satisfy the above two constraints and are the eigenfunctions of the LCT with parameters $\left\{-\sigma^{-1}, 0,0,-\sigma\right\}$, they are too complicated, and we will not discuss them here.

With the aid of above results and Property B described in Section III-A, we can derive the eigenfunctions of the LCT for the cases where $a+d>2$ and $a+d<-2$.

\section{B. Case Where $a+d>2$}

For the case where $a+d>2$, we choose $\left\{a_{2}, b_{2}, c_{2}, d_{2}\right\}$ as $\left\{\sigma^{-1}, 0,0, \sigma\right\}$ in (29). Then, because $a+d=a_{2}+d_{2}$ must be satisfied

$$
a+d=\sigma+\frac{1}{\sigma} .
$$

Solving the above equality, we obtain

$$
\sigma=\frac{a+d \pm \sqrt{(a+d)^{2}-4}}{2} .
$$

Then, after substituting $\left\{a_{2}, b_{2}, c_{2}, d_{2}\right\}=\left\{\sigma^{-1}, 0,0, \sigma\right\}$ into (27), we obtain

$$
\begin{aligned}
{\left[\begin{array}{ll}
a & b \\
c & d
\end{array}\right] } & =\left[\begin{array}{ll}
a_{1} & b_{1} \\
c_{1} & d_{1}
\end{array}\right]\left[\begin{array}{cc}
\sigma^{-1} & 0 \\
0 & \sigma
\end{array}\right]\left[\begin{array}{cc}
d_{1} & -b_{1} \\
-c_{1} & a_{1}
\end{array}\right] \\
& =\left[\begin{array}{ll}
\frac{a_{1} d_{1}}{\sigma}-b_{1} c_{1} \sigma & -\frac{a_{1} b_{1}}{\sigma}+a_{1} b_{1} \sigma \\
\frac{c_{1} d_{1}}{\sigma}-c_{1} d_{1} \sigma & -\frac{b_{1} c_{1}}{\sigma}+a_{1} d_{1} \sigma
\end{array}\right] .
\end{aligned}
$$

The general solution of the above equation is

$$
\begin{gathered}
a_{1}: \text { free to choose, except } 0 \\
b_{1}=\frac{s \cdot b}{a_{1} \sqrt{(a+d)^{2}-4}} \\
c_{1}=\frac{-2 a_{1} \cdot s \cdot c}{s(d-a)+\sqrt{(a+d)^{2}-4}} \\
d_{1}=\frac{1}{2 a_{1}}\left(\frac{s \cdot(d-a)}{\sqrt{(a+d)^{2}-4}}+1\right)
\end{gathered}
$$

where $s=\operatorname{sgn}\left(\sigma-\sigma^{-1}\right)$. Then, together with Property B in Section III-A, we can conclude that when $a+d>2$, if $f(t)$ is the scaling invariant function with scaling ratio $1 / \sigma$ and eigenvalue $\lambda$, i.e.,

$$
\sqrt{\sigma} \cdot f(\sigma \cdot t)=\lambda \cdot f(t)
$$

then

$$
\phi_{F}(t)=O_{F}^{\left(a_{1}, b_{1}, c_{1}, d_{1}\right)}(f(t))
$$

will be the eigenfunction of the LCT, where $\sigma,\left\{a_{1}, b_{1}, c_{1}, d_{1}\right\}$ are calculated from (121) and (123), and the corresponding eigenvalue is also $\lambda$.

We also notice that when the value of $\left\{a_{1}, b_{1}, c_{1}, d_{1}\right\}$ is (123), then

$$
\begin{aligned}
{\left[\begin{array}{ll}
a_{1} & b_{1} \\
c_{1} & d_{1}
\end{array}\right]=} & {\left[\begin{array}{cc}
1 & \frac{s \cdot b}{\sqrt{(a+d)^{2}-4}} \\
\frac{-2 s \cdot c}{s(d-a)+\sqrt{(a+d)^{2}-4}} & \frac{1}{2}\left(\frac{s \cdot(d-a)}{\sqrt{(a+d)^{2}-4}}+1\right)
\end{array}\right] } \\
& \cdot\left[\begin{array}{cc}
a_{1} & 0 \\
0 & a_{1}^{-1}
\end{array}\right] \\
{\left[\begin{array}{ll}
a_{1} & b_{1} \\
c_{1} & d_{1}
\end{array}\right]=} & {\left[\begin{array}{ll}
1 & 0 \\
\tau & 1
\end{array}\right] \cdot\left[\begin{array}{cc}
1 & \eta \\
0 & 1
\end{array}\right] \cdot\left[\begin{array}{cc}
a_{1} & 0 \\
0 & a_{1}^{-1}
\end{array}\right] }
\end{aligned}
$$

where

$$
\tau=\frac{-2 s \cdot c}{s(d-a)+\sqrt{(a+d)^{2}-4}}, \quad \eta=\frac{s \cdot b}{\sqrt{(a+d)^{2}-4}} .
$$

Therefore, the eigenfunctions of the LCT when $a+d>2$ shown in (125) can be rewritten as

$$
\begin{aligned}
\phi_{F}(t)= & O_{F}^{\left(a_{1}, b_{1}, c_{1}, d_{1}\right)}(f(t)) \\
= & O_{F}^{(1,0, \tau, 1)}\left(O_{F}^{(1, \eta, 0,1)}\left(\sqrt{a_{1}^{-1}} \cdot f\left(a_{1}^{-1} t\right)\right)\right) \\
= & \frac{1}{\sqrt{j 2 \pi \eta a_{1}}} e^{(j / 2) \cdot \tau \cdot t^{2}} \\
& \cdot \int_{-\infty}^{\infty} e^{(j / 2 \eta) \cdot(t-x)^{2}} \cdot f\left(a_{1}^{-1} x\right) \cdot d x
\end{aligned}
$$

We can then set

$$
g(t)=f\left(a_{1}^{-1} t\right) .
$$

From (124), we can prove that

$$
\sqrt{\sigma} \cdot g(\sigma \cdot t)=\lambda \cdot g(t)
$$

must be satisfied. Therefore, no matter what the value of $a_{1}$ is, $g(t)=f\left(a_{1}^{-1} t\right)$ is always a scaling invariant function with scaling ratio $1 / \sigma$, so the parameter $a_{1}$ essentially has no effect. Thus, the results in (121)-(125) can be simplified. We summarize the simplified results in the following.

In the case where $a+d>2$, if $g(t)$ is a scaling invariant function with scaling ratio $1 / \sigma$ and eigenvalue $\lambda$

$$
\begin{aligned}
\sqrt{\sigma} \cdot g(\sigma \cdot t) & =\lambda \cdot g(t) \\
\text { where } \sigma & =\frac{a+d \pm \sqrt{(a+d)^{2}-4}}{2}
\end{aligned}
$$

then

$$
\begin{aligned}
\phi_{F}^{(\tau, \eta, \sigma)}(t)=\frac{1}{\sqrt{j 2 \pi \eta}} \cdot e^{(j / 2) \cdot \tau \cdot t^{2}} \\
\cdot \int_{-\infty}^{\infty} e^{(j / 2 \eta) \cdot(t-x)^{2}} \cdot g(x) \cdot d x
\end{aligned}
$$


where

$$
\begin{aligned}
& \tau=\frac{-2 s \cdot c}{s(d-a)+\sqrt{(a+d)^{2}-4}}, \\
& \eta=\frac{s \cdot b}{\sqrt{(a+d)^{2}-4}}, \quad s=\operatorname{sgn}\left(\sigma-\sigma^{-1}\right)
\end{aligned}
$$

will be the eigenfunction of the LCT, and the corresponding eigenvalue is also $\lambda$.

In (132), there are many possible choices for $g(t)$. We can choose $g(t)$ as the functions developed by the fractal theory, or the functions listed in (115)-(118), or other scaling-invariant functions. In fact, all the functions that are scaling-invariant to the scaling ratio of $1 / \sigma$ can be used here. For example, in (132), we can choose $g(t)$ as delta function $\delta(t)$ (we remember that the delta function is also a scaling-invariant function). Then, (132) becomes

$$
\phi_{F}^{(\tau, \eta, \sigma)}(t)=\sqrt{\frac{1}{2 \pi}} \cdot e^{(j / 4 b) \cdot\left(d-a \pm \sqrt{(a+d)^{2}-4}\right) \cdot t^{2}} .
$$

Here, we have normalized the result and ignored some constant phase. This chirp function is indeed the eigenfunction of the LCT when $a+d>2$.

From (132), we can conclude that the eigenfunctions of the LCT, when $a+d>2$, are the chirp multiplication, chirp convolution of scaling-invariant functions. This is especially true when

$$
b=0 .
$$

Then, from (97) and (133)

$$
\eta=0, \quad \lim _{\eta \rightarrow 0} e^{(j / 2 \eta) \cdot(t-x)^{2}}=\sqrt{j 2 \pi \eta} \cdot \delta(t-x)
$$

ad therefore, the chirp convolution in (132) can be saved. In the case, where

$$
c=0
$$

since $\tau=0$, the chirp multiplication in (132) can be saved.

We note, because for the scaling operations, all the eigenvalues are possible, all the eigenvalues are also possible for the LCT in the case where $a+d>2$. We remember that for the cases where $|a+d|<2$ and $|a+d|=2$, the eigenvalues of the LCT always have the unity amplitude, but for the case where $a+d>2$, it is possible that the amplitudes of the eigenvalues of the LCT are not equal to 1. For example, if in (132) we choose $g(t)$ as a constant function or step function, then the eigenvalue will be $\lambda=\sqrt{\sigma}$, and if we choose $g(t)$ as $t^{n}$, then the eigenvalue will be $\lambda=\sigma^{n+(1 / 2)}$. From the power preservation property of the LCT

$$
\begin{aligned}
\int_{-\infty}^{\infty}\left|F_{(a, b, c, d)}(u)\right|^{2} d u & =\int_{-\infty}^{\infty}|f(t)|^{2} d t \\
\text { where } F_{(a, b, c, d)}(u) & =O_{F}^{(a, b, c, d)}(f(t))
\end{aligned}
$$

it seems that the eigenvalues of the LCT must have unity amplitude, but in fact, (138) is satisfied only for the case where

$$
\int_{-\infty}^{\infty}|f(t)|^{2} \cdot d t<\infty
$$

However, for many of the scaling-invariant functions, such as the functions listed in (115), (116), and (118), the constraint of (139) will not be satisfied. Therefore, the eigenfunctions of the LCT in the case $a+d>2$ will also not satisfy the power preservation property, and their corresponding eigenvalues may not have unity amplitudes.

\section{Case Where $a+d<-2$}

For the case where $a+d<-2$, in (29), we choose $\left\{a_{2}, b_{2}, c_{2}, d_{2}\right\}$ as $\left\{-\sigma^{-1}, 0,0,-\sigma\right\}$. Then, because $a+d=a_{2}+d_{2}$

$$
a+d=-\sigma-\frac{1}{\sigma}
$$

Solving the above equation, we obtain

$$
\sigma=\frac{-a-d \pm \sqrt{(a+d)^{2}-4}}{2}
$$

Then, after substituting $\left\{a_{2}, b_{2}, c_{2}, d_{2}\right\}=\left\{-\sigma^{-1}, 0,0,-\sigma\right\}$ into (27), we obtain

$$
\begin{aligned}
{\left[\begin{array}{ll}
a & b \\
c & d
\end{array}\right] } & =\left[\begin{array}{ll}
a_{1} & b_{1} \\
c_{1} & d_{1}
\end{array}\right]\left[\begin{array}{cc}
-\sigma^{-1} & 0 \\
0 & -\sigma
\end{array}\right]\left[\begin{array}{cc}
d_{1} & -b_{1} \\
-c_{1} & a_{1}
\end{array}\right] \\
& =\left[\begin{array}{ll}
-\frac{a_{1} d_{1}}{\sigma}+b_{1} c_{1} \sigma & \frac{a_{1} b_{1}}{\sigma}-a_{1} b_{1} \sigma \\
-\frac{c_{1} d_{1}}{\sigma}+c_{1} d_{1} \sigma & \frac{b_{1} c_{1}}{\sigma}-a_{1} d_{1} \sigma
\end{array}\right] .
\end{aligned}
$$

The general solution of the above equation is

$$
\begin{aligned}
& a_{1}: \text { free to choose, except } 0 \\
& b_{1}=\frac{s \cdot b}{a_{1} \sqrt{(a+d)^{2}-4}} \\
& c_{1}=\frac{-2 a_{1} \cdot s \cdot c}{s(d-a)+\sqrt{(a+d)^{2}-4}} \\
& d_{1}=\frac{1}{2 a_{1}}\left(\frac{s \cdot(d-a)}{\sqrt{(a+d)^{2}-4}}+1\right)
\end{aligned}
$$

where

$$
s=\operatorname{sgn}\left(\sigma^{-1}-\sigma\right) .
$$

This is the same as (123), except that the definition of $s$ is changed. Thus, with a little modification, the results in Section V-B can also be applied here.

From the similar process to derive (131)-(133), we find, if $g(t)$ is a symmetric (or anti-symmetric) scaling-invariant function

$$
\begin{aligned}
\sqrt{-\sigma} \cdot g(-\sigma \cdot t) & =\lambda \cdot g(t) \\
\text { where } \sigma & =\frac{-a-d \pm \sqrt{(a+d)^{2}-4}}{2}
\end{aligned}
$$


then

$$
\begin{aligned}
\phi_{G}^{(\tau, \eta, \sigma)}(t)=\frac{1}{\sqrt{j 2 \pi \eta}} \cdot e^{(j / 2) \cdot \tau \cdot t^{2}} & \cdot \int_{-\infty}^{\infty} e^{(j / 2 \eta) \cdot(t-x)^{2}} \cdot g(x) \cdot d x
\end{aligned}
$$

where

$$
\begin{aligned}
& \tau=\frac{-2 s \cdot c}{s(d-a)+\sqrt{(a+d)^{2}-4}} \\
& \eta=\frac{s \cdot b}{\sqrt{(a+d)^{2}-4}}, \quad s=\operatorname{sgn}\left(\sigma^{-1}-\sigma\right)
\end{aligned}
$$

will be the eigenfunction of the LCT when $a+d<-2$, and the corresponding eigenvalue is also $\lambda$. There are many choices for $g(t)$. For example, the constant function, delta function, $t^{n}$, and all the symmetric/anti-symmetric fractal functions with scaling ratio $\sigma$ can be used as $g(t)$ to derive the eigenfunctions of the LCT when $a+d<-2$.

Thus, from (146), the eigenfunctions of the LCT when $a+$ $d<-2$ are the chirp multiplication, chirp convolution of symmetric (or anti-symmetric) scaling-invariant functions. As in the case where $a+d>2$, in some special cases, the eigenfunctions of the LCT when $a+d<-2$ can be simplified. When $b=0$, the chirp convolution in (146) can be saved, and in the case where $c=0$, the chirp multiplication can be saved in (146).

As in the case where $a+d>2$, all the values of the eigenvalues are possible for the case where $a+d<-2$. The amplitudes of eigenvalues of the LCT when $a+d<-2$ are not constrained to be 1 .

We also notice that, as in the case of $|a+d|=2$, when $|a+d|>2$, there are also infinite complete linear independent eigenfunctions sets for the LCT.

\section{APPlications FOR SElf-IMAgING PROBlemS}

From the current research works about the self-functions of fractional Fourier transform (FRFT), there are mainly three applications of the eigenfunctions of FRFT:

1) self-imaging problems;

2) resonance problems;

3) mode selection.

Similarly, we can also use the eigenfunctions of the LCT we derive for these applications.

In this section, we will discuss how to use the eigenfunctions of the LCT to analyze the self-imaging problems of optical system. Before discussing this topic, we first review how to use the LCT to represent the optical systems briefly and introduce an important property.

\section{A. Relations Between the LCT and Optical System}

The LCT has very intimated relations with optics because many of the wave propagation operations in optics can be expressed as the special case of the LCT [13]-[15]. For example, from (14), we can use the LCT with parameters $\{a, b, c, d\}=\{1, z \lambda / 2 \pi, 0,1\}$ to represent the monochromic light propagating through the free space with distance $z$.
Besides, the monochromic light with wavelength $\lambda$ propagating through the cylinder lens with focal length $f$ can be expressed as

$$
\begin{aligned}
O_{\text {lens }}^{f}(g(x))= & e^{j(\pi / \lambda) n \Delta} e^{-j(\pi / f \cdot \lambda) x^{2}} g(x) \\
& n: \text { refractive index, } \Delta: \text { thickness of lens. }
\end{aligned}
$$

It just corresponds to the LCT with parameters $\{1,0,-2 \pi / f \lambda, 1\}$

$$
O_{\text {lens }}^{f}(g(x))=e^{j(\pi / \lambda) n \Delta} O_{F}^{(1,0,-2 \pi / f \lambda, 1)}(g(x)) .
$$

From (14) and (149) and the fact that the combination of optical components can be represented by the product of the $a b c d$ matrices that represent the LCTs [see (8)], if an optical system just has free spaces and lenses, then we can use the LCT to represent it. For example, if an optical system contains a lens with focal length $f$ followed by a free space with length $z$, then since

$$
\left[\begin{array}{cc}
1 & \frac{z \lambda}{2 \pi} \\
0 & 1
\end{array}\right]\left[\begin{array}{cc}
1 & 0 \\
\frac{-2 \pi}{f \lambda} & 1
\end{array}\right]=\left[\begin{array}{cc}
1-\frac{z}{f} & \frac{z \lambda}{2 \pi} \\
\frac{-2 \pi}{f \lambda} & 1
\end{array}\right]
$$

we can use the LCT with parameters $\{1-z / f, z \lambda / 2 \pi$, $-2 \pi / f \lambda, 1\}$ to represent this optical system.

We then describe an important property. This property is very helpful for discussing the self-imaging phenomena of optical system.

(Property C) Conditions That two LCTs Are Equivalent in Optics [29]: Because when $a_{1} / a_{2}=b_{1} / b_{2}$, the transform results of the LCTs with parameters $\left\{a_{1}, b_{1}, c_{1}, d_{1}\right\}$ and $\left\{a_{2}, b_{2}, c_{2}, d_{2}\right\}$ will have the relation as follows:

$$
\begin{aligned}
\left|F_{\left(a_{2}, b_{2}, c_{2}, d_{2}\right)}(u)\right| & =\left|\sqrt{\sigma} \cdot F_{\left(a_{1}, b_{1}, c_{1}, d_{1}\right)}(\sigma \cdot u)\right| \\
\text { where } \sigma & =\frac{a_{1}}{a_{2}}=\frac{b_{1}}{b_{2}} .
\end{aligned}
$$

Therefore, we can conclude the following.

a) If only the intensity is considered, then two LCTs are equivalent if

$$
a_{1}=a_{2}, \quad b_{1}=b_{2} \text {. }
$$

b) If the difference of scaling is ignored, then two LCTs are equivalent if

$$
a_{1}: b_{1}=a_{2}: b_{2}, \quad c_{1}: d_{1}=c_{2}: d_{2} \text {. }
$$

c) If only the intensity is considered, and the difference of scaling is ignored, then two LCTs are equivalent if

$$
a_{1}: b_{1}=a_{2}: b_{2}
$$

Because, in optics, the difference of phase and scaling are usually ignored, from Property $\mathrm{C}$, the constraints for the input functions to cause the self-imaging phenomena are very relaxed. 
Therefore, for most of the optical systems, there are usually varieties of input functions that can cause the self-imaging phenomena. This will be seen in Section VI-B.

\section{B. Solving the Self-Imaging Problems}

By the relations between the LCT and optical system, we can use the eigenfunctions of the LCT to study the self-imaging phenomena of optical system, i.e., searching the input images that will have the output the same as the input images. If an optical system is the combination of free spaces and lenses, then from the discussion in Section VI-A, we can represent it by the LCT with some parameters $\left\{a_{1}, b_{1}, c_{1}, d_{1}\right\}$. Then, if the input light has the distribution the same as the eigenfunctions of the LCT with parameters $\left\{a_{1}, b_{1}, c_{1}, d_{1}\right\}$, it will cause the self-imaging phenomena for this optical system.

In optics, however, we always ignore the difference of phase, and when discussing the self-imaging phenomena, the difference of scaling is usually tolerable. Therefore, if an optical system can be represented by the LCT with parameters $\left\{a_{1}, b_{1}, c_{1}, d_{1}\right\}$, and then, the eigenfunctions of the LCT with parameters $\left\{a_{1}, b_{1}, c_{1}, d_{1}\right\}$ are not the only possible inputs that can cause the self-imaging phenomena. In fact, from Property $\mathrm{C}$, all the eigenfunctions of the LCT with parameters $\{a, b, c, d\}$ that satisfy $a_{1}: b_{1}=a_{2}: b_{2}$ can also cause the self-imaging phenomena. Therefore, when discussing the self-imaging phenomena of an optical system, we can use the algorithm as follows.

1) Find the parameters $\left\{a_{1}, b_{1}, c_{1}, d_{1}\right\}$ of the LCT to represent this optical system.

2) If the difference of scaling is considered, then we can follow the algorithm.

i) Always fix $a, b$ as $a=a_{1}, b=b_{1}$.

ii) Vary $d$ from $-\infty$ to $\infty$ in iterative, and set $c$ as $(a d-$ 1) $/ b$.

Then, all the parameters sets $\{a, b, c, d\}$ of the LCT that satisfy $a=a_{1}, b=b_{1}$ can be found. The eigenfunctions of the LCTs with all the parameters set $\{a, b, c, d\}$ found above can all cause the self-imaging phenomena without scaling.

3) If the difference of scaling is ignored, then we can follow the algorithm.

i) Set $a=a_{1} / \sigma, b=b_{1} / \sigma$.

ii) Vary $d$ from $-\infty$ to $\infty$ iteratively and set $c$ as $(a d-1) / b$ to find all the parameters sets $\{a, b, c, d\}$ of the LCT that satisfy $a=a_{1} / \sigma, b=b_{1} / \sigma$.

iii) Vary $\sigma$ from $-\infty$ to $\infty$, and repeat i) and ii) iteratively.

Then, all the parameter sets $\{a, b, c, d\}$ of the LCT that satisfy $a: b=a_{1}: b_{1}$ can be found. The eigenfunctions of the LCTs with all the parameters set $\{a, b, c, d\}$ found above can all cause the self-imaging phenomena when the difference of scaling is ignored, and the scaling ratio (i.e., the ratio $\sigma$ such that $\left|f_{o}(t)\right|=\tau \cdot\left|f_{\mathrm{i}}(\sigma \cdot t)\right|$ where $f_{\mathrm{i}}(t)$ is the input and $f_{o}(t)$ is the output) is

$$
\sigma=\frac{a_{1}}{a}=\frac{b_{1}}{b}
$$

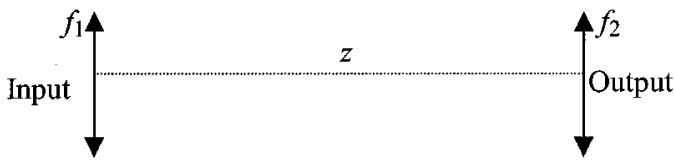

Fig. 2. Optical system with two lenses and one free space.

We give the following example. For the optical system in Fig. 2, we can represent it by the LCT with the parameters as follows:

$$
\begin{aligned}
{\left[\begin{array}{cc}
1 & 0 \\
-\frac{2 \pi}{f_{2} \lambda} & 1
\end{array}\right]\left[\begin{array}{cc}
1 & \frac{z \lambda}{2 \pi} \\
0 & 1
\end{array}\right]\left[\begin{array}{cc}
1 & 0 \\
-\frac{2 \pi}{f_{1} \lambda} & 1
\end{array}\right] } \\
=\left[\begin{array}{cc}
1-\frac{z}{f_{1}} & \frac{z \lambda}{2 \pi} \\
-\frac{2 \pi}{f_{1} \lambda}-\frac{2 \pi}{f_{2} \lambda}+\frac{2 \pi z}{f_{1} f_{2} \lambda} & 1-\frac{z}{f_{2}}
\end{array}\right] .
\end{aligned}
$$

Therefore, all the eigenfunctions of the LCT with the parameters $\{a, b, c, d\}$ where

$$
a: b=1-\frac{z}{f_{1}}: \frac{z \lambda}{2 \pi}
$$

will cause the self-imaging phenomena for this optical system, and the scaling ratio is

$$
\sigma=\frac{1-\frac{z}{f_{1}}}{a}=\frac{z \lambda}{2 \pi b}
$$

When

$$
a=1-\frac{z}{f_{1}}, \quad b=\frac{z \lambda}{2 \pi}
$$

then $\sigma=1$, and the eigenfunctions of the LCT in this case will cause the self-imaging phenomena without scaling difference.

In the following, we will just discuss the case where $\sigma=1$, i.e., no scaling difference. We can apply the algorithm introduced in this subsection. We first fix $a$ and $b$ as (158).

The value of $a+d$ determines the form of the eigenfunctions of the LCT. Since $d$ can be any value, and

$$
a+d=1-\frac{z}{f_{1}}+d
$$

the value of $a+d$ can also be any value. Varying the values of $d$ from $-\infty$ to $\infty$, we can find all the possible inputs that will cause the self-imaging phenomena without scaling difference for the optical system in Fig. 2.

1) The case where $d<-3+z / f_{1}$ (i.e., $a+d<-2$ ).

In this case, from Section V-C, we will find that the functions defined as (146) (i.e., the chirp multiplication, chirp convolution of the symmetric/anti-symmetric self-similar functions) will cause the self-imaging phenomena without scaling. The value of $\sigma, \tau$, and $\eta$ can be calculated from (145) and (147) by substituting $\{a, b, c, d\}$ as $\left\{1-z / f_{1}, z \lambda / 2 \pi, c, d\right\}$.

2) The case where $d=-3+z / f_{1}$ (i.e., $a+d=-2$ ) (suppose $\left.z \neq 2 f_{1}\right)$. 
TABLE I

EIGENFUNCTIONS OF the LCT FOR ALL THE CASES

\begin{tabular}{|c|c|c|}
\hline & Eigenfunctions & Eigenvalues \\
\hline $\begin{array}{l}\text { Case A } \\
(|a+d|<2)\end{array}$ & $\begin{array}{l}\frac{1}{\sqrt{\sigma \cdot 2^{m} m ! \sqrt{\pi}}} \exp \left(\frac{-(1+i \tau) t^{2}}{2 \sigma^{2}}\right) H_{m}\left(\frac{t}{\sigma}\right) \\
\left.\text { where } H_{m}(t): \text { Hermite function (see }(18)\right) \text {, } \\
\qquad \sigma^{2}=\frac{2|b|}{\sqrt{4-(a+d)^{2}}}, \tau=\frac{\operatorname{sgn}(b) \cdot(a-d)}{\sqrt{4-(a+d)^{2}}} .\end{array}$ & $\begin{array}{l}\left(e^{-j \alpha}\right)^{1 / 2} \exp (-i \alpha m) \\
\text { where } \alpha=\cos ^{-1}\left(\frac{a+d}{2}\right) \\
=\sin ^{-1}\left(\frac{\operatorname{sgn}(b)}{2} \sqrt{4-(a+d)^{2}}\right.\end{array}$ \\
\hline $\begin{array}{l}\text { Case B } \\
\begin{array}{c}(a+d=2 \\
b=0)\end{array}\end{array}$ & $\begin{array}{l}\sqrt{E^{-1}} \cdot\left[\sum_{n=0}^{\infty} A_{n} \delta\left(t-\sqrt{4 n \pi|c|^{-1}+h}\right)+\right. \\
\left.\quad \sum_{m=0}^{\infty} B_{m} \delta\left(t+\sqrt{4 m \pi|c|^{-1}+h}\right)\right] \\
\text { where } 0 \leq h<4 \pi /|c|, A_{n} \text { 's, } B_{m} \text { 's are free to choose, } \\
\qquad E=\sum_{n=0}^{\infty}\left(\left|A_{n}\right|^{2}+\left|B_{n}\right|^{2}\right)\end{array}$ & $\exp (j c h / 2)$ \\
\hline $\begin{array}{l}\text { Case C } \\
\begin{array}{l}(a+d=-2 \\
b=0)\end{array}\end{array}$ & $\begin{array}{l}\text { The same as case B, except for } A_{n}=B_{n} \text { for all } n \text { or } \\
A_{n}=-B_{n} \text { for all } n \text {. }\end{array}$ & $\pm(-1)^{1 / 2} \exp (j c h / 2)$ \\
\hline $\begin{array}{l}\text { Case D } \\
\begin{array}{c}(a+d=2 \\
b \neq 0)\end{array}\end{array}$ & $\begin{array}{c}\sqrt{\frac{1}{j 2 \pi \rho}} \cdot e^{j \frac{d-a}{4 b} t^{2}} \int_{-\infty}^{\infty} e^{j \frac{(t-x)^{2}}{2 \rho}} g(x) d x \\
\text { where } g(t)=\sqrt{\frac{1}{2 \pi S}}\left[\sum_{n=0}^{\infty} C_{n} \exp \left(j t \sqrt{4 n \pi|b|^{-1}+h}\right)+\right. \\
\left.\sum_{m=0}^{\infty} D_{m} \exp \left(-j t \sqrt{4 m \pi|b|^{-1}+h}\right)\right], \\
0 \leq h<4 \pi|b|, C_{n}{ }^{\prime} \mathrm{s}, D_{m} \text { 's are free to choose, } \\
S=\sum_{n=0}^{\infty}\left(\left|C_{n}\right|^{2}+\left|D_{n}\right|^{2}\right)\end{array}$ & $\exp (-j b h / 2)$ \\
\hline $\begin{array}{l}\text { Case E } \\
\begin{array}{l}(a+d=-2 \\
b \neq 0)\end{array}\end{array}$ & $\begin{array}{l}\text { The same as case } \mathrm{B} \text {, except for } C_{n}=D_{n} \text { for all } n \text { or } \\
C_{n}=-D_{n} \text { for all } n \text {. }\end{array}$ & $\pm(-1)^{1 / 2} \exp (-j b h / 2)$ \\
\hline $\begin{array}{l}\text { Case F } \\
(a+d>2)\end{array}$ & $\begin{array}{l}\quad \frac{1}{\sqrt{j 2 \pi \eta}} \cdot e^{\frac{j}{2} \cdot \tau \cdot t^{2}} \int_{-\infty}^{\infty} e^{\frac{j}{2 \eta} \cdot(t-x)^{2}} \cdot g(x) \cdot d x, \\
\text { where } \sqrt{\sigma} \cdot g(\sigma \cdot t)=\lambda \cdot g(t), \\
\qquad \begin{aligned} & \tau=\frac{-2 s \cdot c}{s(d-a)+\sqrt{(a+d)^{2}-4}}, \quad \eta=\frac{s \cdot b}{\sqrt{(a+d)^{2}-4}}, \\
& \quad \sigma=\left(a+d \pm \sqrt{(a+d)^{2}-4}\right) / 2, s=\operatorname{sgn}\left(\sigma-\sigma^{-1}\right) .\end{aligned}\end{array}$ & $\lambda$ (defined as left) \\
\hline $\begin{array}{l}\text { Case } \mathrm{G} \\
(a+d<-2)\end{array}$ & $\begin{array}{l}\text { The same as case } \mathrm{F} \text {, except for } \\
\quad g(t)= \pm g(-t) \\
\text { must also be satisfied, and } s \text { is changed as } \operatorname{sgn}\left(\sigma^{-1}-\sigma\right) \text {. }\end{array}$ & $\lambda$ \\
\hline
\end{tabular}

In this case, from Section IV-E, we find the functions that follow will cause the self-imaging phenomena without scaling:

$\phi(t)=e^{j \cdot \pi\left(\left(-2+z / f_{1}\right) / z \lambda\right) t^{2}} \cdot \int_{-\infty}^{\infty} e^{j(t-x)^{2} / 2 \rho} g(x) d x$

where

$$
\begin{aligned}
& g(t)=\sum_{n=0}^{\infty} C_{n} \cos \left(2 \pi t \sqrt{\frac{2 n}{z \lambda}+h}\right) \text { or } \\
& g(t)=\sum_{n=0}^{\infty} C_{n} \sin \left(2 \pi t \sqrt{\frac{2 n}{z \lambda}+h}\right)
\end{aligned}
$$

where $\rho$ and $C_{n}$ s are free to choose $0 \leq h<2 / z \lambda$. We can just choose $\rho=0$ so that

$$
\phi(t)=A \cdot e^{j \cdot \pi\left(\left(-2+z / f_{1}\right) / z \lambda\right) t^{2}} \cdot g(t) .
$$

3) The case where $-3+z / f_{1}<d<1+z / f_{1}$, (i.e., $-2<$ $a+d<2$ ).

In this case, from Section III, we find the functions that follow cause the self-imaging phenomena without scaling:

$$
\phi_{m}^{(\sigma, \tau)}(t)=\exp \left(\frac{-(1+i \tau) t^{2}}{2 \sigma^{2}}\right) \cdot H_{m}\left(\frac{t}{\sigma}\right)
$$


where

$$
\sigma^{2}=\frac{z \lambda}{\pi \zeta}, \quad \tau=\frac{1-d-\frac{z}{f_{1}}}{\zeta}, \quad \zeta=\sqrt{4-\left(1+d-\frac{z}{f_{1}}\right)^{2}} .
$$

4) The case where $d=1+z / f_{1}$, (i.e., $a+d=2$ ).

In this case, from Section IV-D, we find the functions that follow will cause the self-imaging phenomena without scaling:

$$
\phi(t)=e^{j\left(\pi / f_{1} \lambda\right) t^{2}} \cdot \int_{-\infty}^{\infty} e^{j(t-x)^{2} / 2 \rho} g(x) d x
$$

where

$$
\begin{aligned}
g(t)=\sum_{n=0}^{\infty} C_{n} \exp & \left(j 2 \pi t \sqrt{\frac{2 n}{z \lambda}+h}\right) \\
& +\sum_{m=0}^{\infty} D_{m} \exp \left(-j 2 \pi t \sqrt{\frac{2 m}{z \lambda}+h}\right)
\end{aligned}
$$

where $\rho, C_{n}$ s, and $D_{m}$ s are free to choose

$$
0 \leq h<\frac{2}{z \lambda} \text {. }
$$

We can just choose $\rho=0$ so that

$$
\phi(t)=e^{j \cdot \pi \cdot t^{2} / f_{1} \lambda} \cdot g(t)
$$

5) The case where $d>1+z / f_{1}$, (i.e., $a+d>2$ ).

In this case, from Section V-B, we will find the functions defined as (132) (i.e., the chirp multiplication, chirp convolution of the self-similar functions) will cause the selfimaging phenomena without scaling, where the value of $\sigma$, $\tau, \eta$ can be calculated from (131) and (133) by substituting $\{a, b, c, d\}$ as $\left\{1-z / f_{1}, z \lambda / 2 \pi, c, d\right\}$.

Thus, there are many types of input images that can cause the self-imaging phenomena without scaling for the optical system as Fig. 2. We notice that the last lens (with focal length $f_{2}$ ) does not have any effect on the self-imaging phenomena.

\section{SUMMARY AND CONCLUSION}

In this paper, we divide the discussions about the eigenfunctions of linear canonical transform (the LCT) into seven cases. We make a summary for the eigenfunctions and eigenvalues of the LCT for each case in Table I.

In this paper, we have derived the eigenfunctions and eigenvalues of linear canonical transform (the LCT) for all the cases. Whether $|a+d|$ is greater than 2 and whether $b$ equals 0 will affect the form of the eigenfunctions of the LCT. We also use the eigenfunctions of the LCT we derive to discuss the self-imaging phenomena. We find that Hermite functions, (almost) periodic functions, self-similar functions (fractals), and the chirp multiplication, chirp convolution of these functions may possibly cause the self-imaging phenomena in optical systems.

Besides, since we can use the eigenfunctions of FRFT to discuss the mode selection problems and the resonance phenomena of radar system and GRIN medium, mode selection and resonance phenomena analysis are also the potential applications of the eigenfunctions of the LCT.

\section{REFERENCES}

[1] K. B. Wolf, "Integral transforms in science and engineering," in Canonical Transform. New York: Plenum, 1979, ch. 9.

[2] S. Abe and J. T. Sheridan, "Optical operations on wave functions as the Abelian subgroups of the special affine Fourier transformation," Opt. Lett., vol. 19, no. 22, pp. 1801-1803, 1994.

[3] L. M. Bernardo, "ABCD matrix formalism of fractional Fourier optics," Opt. Eng., vol. 35, no. 3, pp. 732-740, Mar. 1996.

[4] S. Abe and J. T. Sheridan, "Almost Fourier and almost Fresnel transformation," Opt. Commun., vol. 113, pp. 385-388, 1995.

[5] M. Moshinsky and C. Quesne, "Linear canonical transformations and their unitary representations," J. Math. Phys., vol. 12, no. 8, pp. 1772-1783, Aug. 1971.

[6] S. A. Collins, "Lens-system diffraction integral written in terms of matrix optics," J. Opt. Soc. Amer., vol. 60, pp. 1168-1177, Sept. 1970.

[7] V. Namias, "The fractional order Fourier transform and its application to quantum mechanics," J. Inst. Math. Appl., vol. 25, pp. 241-265, 1980.

[8] L. B. Almeida, "The fractional Fourier transform and time-frequency representations," IEEE Trans. Signal Processing, vol. 42, pp. 3084-3091, Nov. 1994.

[9] H. M. Ozaktas, M. A. Kutay, and Z. Zalevsky, The Fractional Fourier Transform With Applications in Optics and Signal Processing. New York: Wiley, 2000.

[10] J. W. Goodman, Introduction to Fourier Optics, 2nd ed. New York: McGraw-Hill, 1988.

[11] S. C. Pei and J. J. Ding, "Eigenfunctions of the canonical transform and self-imaging problems in optical system," in Proc. IEEE Int. Conf. Acoust., Speech, Signal Process., Istanbul, Turkey, June 2000, pp. $73-76$.

[12] D. F. V. James and G. S. Agarwal, "The generalized Fresnel transform and its applications to optics," Opt. Commun., vol. 126, pp. 207-212, May 1996.

[13] M. J. Bastiaans, "Wigner distribution function and its application to firstorder optics," J. Opt. Soc. Amer., vol. 69, pp. 1710-1716, 1979.

[14] M. Nazarathy and J. Shamir, "First-order optics-A canonical operator representation: Lossless systems," J. Opt. Soc. Amer, vol. 72, pp. 356-364, 1982.

[15] M. J. Bastiaans, "Propagation laws for the second-order moments of the Wigner distribution function in first-order optical systems," Optik, vol. 82, pp. 173-181, 1989.

[16] J. T. Winthrop and C. R. Worthington, "Theory of Fresnel images. 1. Plane periodic objects in monochromatic light," J. Opt. Soc. Amer., vol. 55, pp. 373-381, 1965.

[17] K. Paiorski, "The self-imaging phenomenon and its applications," in Progress in Optics, E. Wolf, Ed. Amsterdam, The Netherlands: NorthHolland, 1989, pt. 1, vol. 27.

[18] W. Zhao and R. M. Rao, "Discrete-time, continuous-dilation construction of self-similar signals and linear scale-invariant systems,", to be published.

[19] G. W. Wornell, Signal Processing With Fractals. Upper Saddle River, NJ: Prentice-Hall, 1996.

[20] R. N. Bracewell, The Fourier Integral and Its Applications, 3rd ed. Boston, MA: McGraw-Hill, 2000.

[21] T. Alieva and A. M. Barbe, "Self-fractional Fourier functions and selection of modes," J. Phys. A: Math. Gen., vol. 30, pp. 211-215, 1997.

[22] - "Self-imaging in fractional Fourier transform systems," Opt. Commun., vol. 152, pp. 11-15, June 1998.

[23] S. G. Lipson and H. Lipson, Optical Physics, 2nd ed. Cambridge, U.K.: Cambridge Univ. Press, 1981, pp. 190-192.

[24] S. J. Leon, Linear Algebra With Applications, 4th ed. Englewood Cliffs, NJ: Prentice-Hall, 1994.

[25] T. Alieva and M. J. Bastiaans, "Powers of transfer matrices determined by means of eigenfunctions," J. Opt. Soc. Amer. A, vol. 16, no. 10, pp. 2413-2418, Oct. 1999.

[26] H. Hamam and J. L. de Bougrenet de la Tocnaye, "Programmable joint fractional Talbot computer-generated holograms," J. Opt. Soc. Amer., vol. 12, no. 2, pp. 314-324, Feb. 1995.

[27] A. W. Lohmann, "An array illuminator based on the Talbot effect," Optik, vol. 79, pp. 41-45, 1988.

[28] J. Leger and G. J. Swanson, "Efficient array illuminator using binaryoptics phase plates as fractional Talbot planes," Opt. Lett., vol. 15, pp. 288-290, 1990.

[29] T. Alieva and F. Agullo-Lopez, "Imaging in first-order optical systems," J. Opt. Soc. Amer. A, vol. 13, no. 12, pp. 2375-2380, Dec. 1996. 


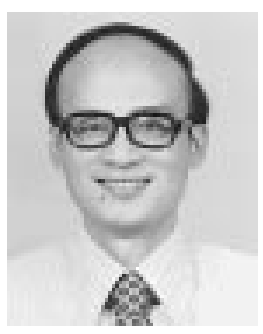

Soo-Chang Pei (F’00) was born in Soo-Auo, Taiwan, R.O.C., in 1949. He received B.S.E.E. degree from National Taiwan University (NTU), Taipei, in 1970 and the M.S.E.E. and Ph.D. degrees from the University of California, Santa Barbara, in 1972 and 1975, respectively.

He was an engineering officer with the Chinese Navy Shipyard from 1970 to 1971. From 1971 to 1975, he was a Research Assistant with the University of California, Santa Barbara. He was Professor and Chairman with the Electrical Engineering Department, Tatung Institute of Technology and NTU, from 1981 to 1983 and 1995 to 1998 , respectively. Presently, he is a Professor with the Eleectrical Engineering Department, NTU. His research interests include digital signal processing, image processing, optical information processing, and laser holography.

Dr. Pei received National Sun Yet-Sen Academic Achievement Award in Engineering in 1984, the Distinguished Research Award from the National Science Council from 1990 to 1998, the Outstanding Electrical Engineering Professor Award from the Chinese Institute of Electrical Engineering in 1998, and the Academic Achievement Award in Engineering from the Ministry of Education in 1998. He has been President of the Chinese Image Processing and Pattern Recognition Society in Taiwan from 1996 to 1998 and is a member of Eta Kappa $\mathrm{Nu}$ and the Optical Society of America.

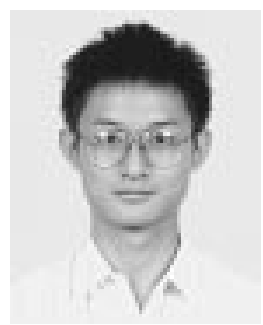

Jian-Jiun Ding was born in 1973 in Pingdong, Taiwan, R.O.C. He received both the B.S. and M.S. degrees in electrical engineering from National Taiwan University (NTU), Taipei, in 1995 and 1997, respectively. $\mathrm{He}$ is currently pursuing the Ph.D. degree under the supervision of Prof. S.-C. Pei with the Department of Electrical Engineering, NTU. He is also a Teaching Assistant at NTU. His current research areas include fractional and affine Fourier transforms, other fractional transforms, orthogonal polynomials, integer transforms, the quaternion Fourier transform, pattern recognition, fractals, filter design, etc. 